TRANSACTIONS OF THE

AMERICAN MATHEMATICAL SOCIETY

Volume 362, Number 2, February 2010, Pages 777-813

S 0002-9947(09)04910-1

Article electronically published on September 11, 2009

\title{
WORKING WITH STRONG REDUCIBILITIES ABOVE TOTALLY $\omega$-C.E. AND ARRAY COMPUTABLE DEGREES
}

\author{
GEORGE BARMPALIAS, ROD DOWNEY, AND NOAM GREENBERG
}

\begin{abstract}
We investigate the connections between the complexity of a c.e. set, as calibrated by its strength as an oracle for Turing computations of functions in the Ershov hierarchy, and how strong reducibilities allow us to compute such sets. For example, we prove that a c.e. degree is totally $\omega$-c.e. iff every set in it is weak truth-table reducible to a hypersimple, or ranked, set. We also show that a c.e. degree is array computable iff every left-c.e. real of that degree is reducible in a computable Lipschitz way to a random left-c.e. real (an $\Omega$-number).
\end{abstract}

\section{INTRODUCTION}

Whilst this paper is underpinned by a number of technical results, its principal goal is to bring into focus a new and potentially important programme in computability theory, in particular, computability theory applied to algorithmic randomness and computable model theory. As we will see, this paper will demonstrate interactions between a number of distinct areas of computability theory, including strong reducibilities, Turing degrees on the computably enumerable sets, dynamic and static properties of computably enumerable sets, various natural classes of degrees, algorithmic randomness, $\Pi_{1}^{0}$ classes and their ranked points, and computable model theory. We mention that the classes of degrees discussed include the totally $\omega$-c.e. degrees and the array computable degrees 11 classes which have had significant recent interest and have deep connections with algorithmic randomness as witnessed by, for example, Kummer 29, Downey and Greenberg [14, and several other articles (see Downey and Hirschfeldt [16]).

Traditionally, one classifies classes of Turing degrees by investigating their computational power as oracles, as is reflected by the structure of the Turing degrees below the degree in question. There are very well-known classical examples of this phenomenon. For example, a c.e. set $A$ is high if it is indistinguishable from the halting problem as measured by the jump operator, in that $A^{\prime} \equiv_{T} \emptyset^{\prime \prime}$. Used as oracles, we would expect that high sets should have computational power similar to that of $\emptyset^{\prime}$ and this intuition is justified by results such as the coincidence of the

Received by the editors December 19, 2007.

2000 Mathematics Subject Classification. Primary 03D25, 03D30.

We wish to thank Frank Stephan for his permission to include Theorem 1.12 and its proof. All authors were supported by the Marsden fund of New Zealand.

${ }^{1}$ Precise definitions will soon follow. 
high degrees with the degrees which contain maximal c.e. sets Thus, high sets have enough computational power to enable us to carry out the construction of a maximal set. Another illustration comes from lowness properties, where $L$ is low if $L^{\prime} \equiv_{T} \emptyset^{\prime}$. Now in terms of the jump operator, low sets are indistinguishable from computable sets. Tying this to our example above, no low c.e. set can be maximal.

The focus of this paper is not on what can be performed below some degree, but the dual question: how complicated is it to compute a set outside a given class, and so how do degrees in these classes behave with respect to degrees above them? A nice example in algorithmic randomness comes from the work on algorithmic lowness, where it has been shown that the only sets $D$ so computationally feeble that there is an $R \geqslant_{T} D$ which is random relative to $D$ are precisely the class of " $K$-trivial" reals. The precise definitions here are not important to our task at hand, but it suffices to say that this class is an important subclass of the low sets, whose members are low in terms of their initial segment complexity (more details can be found in Nies [32]).

For our paper, we begin with the following examples of totally $\omega$-c.e. degrees and their uniform analogue, the array computable degrees. To make the definitions precise, recall that Shoenfield's limit lemma states that a function $f: \omega \rightarrow \omega$ is computable from the degree $\mathbf{0}^{\prime}$ of the halting problem if and only if it has a computable approximation: there is a uniformly computable sequence of functions $\left\langle f_{s}\right\rangle$ which converge pointwise to $f$ (under the discrete topology on $\omega$ ). That is, for all $n$, the mind-change set

$$
\left\{s: f_{s}(n) \neq f_{s+1}(n)\right\}
$$

is finite, and $f_{s}(n)=f(n)$ for almost all $s$. Every computable approximation $\left\langle f_{s}\right\rangle$ gives rise to the mind-change function:

$$
m_{f}(n)=\#\left\{s: f_{s}(n) \neq f_{s+1}(n)\right\} .
$$

Following Ershov, we classify the complexity of a $\Delta_{2}^{0}$ function by how quickly a computable approximation for $f$ can settle; that is, what kind of functions dominate the mind-change function of some approximation for $f$. An order is a non-decreasing and unbounded function; we say that a function $f$ is $h$-c.e. if it has some computable approximation such that $h$ bounds the mind-change function of that approximation. Finally, we say that a function $f$ is $\omega$-c.e. if it is $h$-c.e. for some computable order $h$.

Array computability and total $\omega$-c.e.ness are defined as notions of oracular weakness, in the sense that the definitions say that all functions that can be computed by the degree are simple in the sense that they have reasonable approximations. A c.e. degree $\mathbf{d}$ is array computable if there is some computable order $h$ such that every $f \leqslant_{T} \mathbf{d}$ is $h$-c.e. This notion was defined and investigated by Downey, Jockusch and Stob [19], who showed that the array computable c.e. degrees are exactly the c.e. degrees for which a certain notion of permitting (called "multiple permitting") fails, with immediate consequences for the computational power of $\mathbf{d}$. For example, they showed that a c.e. degree is array non-computable iff it computes a Martin-Pour-El theory. Since it is again not important to our results, we will simply mention that a Martin-Pour-El theory is a special type of an essentially undecidable axiomatizable theory with very few axiomatizable extensions, and it is an analog of a maximal

\footnotetext{
${ }^{2}$ Recall that a co-infinite set $M$ is maximal if for all c.e. $W \supset M$ either $W-M$ is finite or $\omega-W$ is finite.
} 
set for theories. Thus we see that the array non-computable degrees have precisely the power to compute these objects.

Later, this notion of array non-computability was expanded in [21] to the degrees in general by replacing approximations with domination; a Turing degree $\mathbf{d}$ is array computable if there is some $\omega$-c.e. function which dominates all functions computable in $\mathbf{d}$. It was shown in [21] that every array non-computable degree is the join of a minimal pair, and a degree is array non-computable iff it bounds a $p b$-generic real, which is defined as a real which is Cohen generic for dense sets which can be accessed by primitive recursive functions.

The class of totally w-c.e. degrees was defined and investigated by Downey, Greenberg and Weber in 15 and later in 12 . These are the c.e. degrees $\mathbf{d}$ such that every function $f \leqslant_{T} \mathbf{d}$ is $\omega$-c.e. In [15, methods of permitting and anti-permitting arguments were developed for the class of totally $\omega$-c.e. degrees and it was shown that a c.e. degree is not totally $\omega$-c.e. iff it bounds a critical triple (in the c.e. degrees). Here a critical triple is a configuration in the c.e. degrees representing a certain blockage for embeddings. To wit, $\mathbf{a}, \mathbf{b}, \mathbf{c}$ form a critical triple iff $\mathbf{b} \cup \mathbf{a}=\mathbf{b} \cup \mathbf{c}$ and for all $\mathbf{d} \leqslant \mathbf{a}, \mathbf{c}, \mathbf{d} \leqslant \mathbf{b}$. Notice that this gives a natural definition of the totally $\omega$-c.e. degrees in the c.e. degrees. A generalisation of the notion of totally $\omega$-c.e. degree to the degrees has not yet been investigated, but we mention that it is true that a c.e. degree $\mathbf{d}$ is totally $\omega$-c.e. iff every $f \leqslant_{T} \mathbf{d}$ is dominated by some $\omega$-c.e. function.

Both classes are defined in terms of strength as an oracle for computations, and such was the flavour of the results we mentioned about highness and lowness. We focus on the dual question for these classes: how do these classes behave with respect to degrees above them? A result of this type is that array non-computable degrees join to every degree above them (Downey, Jockusch and Stob 21]) and so cannot have strong minimal covers; Ishmukhametov [24] improved this to show that the c.e. degrees which have strong minimal covers are exactly the array computable ones.

In this paper we continue this line of investigation. We tie these classes with another theme that has been central in the investigation of the array computable and totally $\omega$-c.e. degrees: the interplay between Turing and stronger reducibilities, such as weak truth-table reducibility. Recall that a wtt-functional is a Turing functional $\Gamma$ which is accompanied by a computable function $\gamma$ which bounds the use of $\Gamma$-computations; in other words, for all $(\sigma, \tau) \in \Gamma$ (which expresses that if $\sigma$ is an initial segment of an oracle $X$, then $\tau$ is an initial segment of $\Gamma^{X}$ ) we have $|\sigma| \leqslant \gamma(|\tau|)$. For two sets $A, B$ of natural numbers, $A \leqslant{ }_{\text {wtt }} B$ if there is a wtt-functional $\Gamma$ such that $\Gamma^{B}=A$.

The fact that a function is $\omega$-c.e. iff it is wtt-reducible to the halting problem signaled an interesting relationship between the weakness notions in the Turing degrees and weak truth-table reducibility. For example, a c.e. degree is not totally $\omega$-c.e. iff it computes a wtt-triple, i.e. sets $A_{0}, A_{1}$ and $B$ such that $A_{0} \equiv_{T} A_{1}$, $A_{i} \Varangle_{T} B$ but if $C \leqslant_{\mathrm{wtt}} A_{0}, A_{1}$, then $C \leqslant_{\mathrm{wtt}} B$. There are similar results for array computability.

As we mentioned, in this paper we combine weakness notions for Turing degrees, question the effect of weakness for being the result of a computation, rather than its oracle, and put strong reducibilities in the mix. Thus, the main question we ask is: how hard is it for reals in certain classes to strongly compute strong or weak 
reals? Our results express that particular strong reducibilities help understand and characterise our two lowness notions. Thus total $\omega$-c.e.-ness is intimately related with weak truth-table reducibility, and array computability is similarly related to the stronger computable Lipschitz reducibility.

In the first part of the paper, we concern ourselves with weak truth-table reductions and totally $\omega$-c.e. degrees. The first result here was obtained by Chisholm, Chubb, Harizanov, Hirschfeldt, Jockusch, McNicholl and Pingrey [8, who showed that every c.e. degree which is not totally $\omega$-c.e. contains a c.e. set which is not wtt-reducible to any ranked set. Independently, Afshari, Barmpalias, Cooper and Stephan 1 showed that if $\mathbf{d}$ is totally $\omega$-c.e., then every $A \leqslant_{T} \mathbf{d}$ is wtt-reducible to a hypersimple set; but Barmpalias [2, 3] showed that not every c.e. set is wttreducible to a hypersimple set. We prove:

Theorem 1.1. The following are equivalent for a c.e. degree $\mathbf{d}$ :

(1) Every set in $\mathbf{d}$ is wtt-reducible to a ranked set.

(2) Every set in $\mathbf{d}$ is wtt-reducible to a hypersimple set.

(3) Every set in $\mathbf{d}$ is wtt-reducible to a proper initial segment of a computable, scattered linear ordering.

(4) $\mathbf{d}$ is totally $\omega$-c.e.

Moreover, the equivalence still holds if in any of (1), (2) or (3), "set" is replaced by "c.e. set".

We recall the definitions. A set is ranked if it is an element of some countable $\Pi_{1}^{0}$ class (and so it has a Cantor-Bendixson rank). A linear ordering is scattered if it doesn't contain a copy of the rationals (and so repeating the Hausdorff derivative leaves an empty kernel at the end). A set $A \subset \omega$ is hyperimmune if it is infinite, and whenever $\left\langle F_{n}\right\rangle$ is a computable sequence of pairwise disjoint finite sets, there is some $n$ such that $F_{n} \cap A$ is empty. Equivalently, the function which maps $n$ to the $n^{\text {th }}$ element of $A$ (by magnitude) is not dominated by any computable function. Finally, a c.e. set is hypersimple if its complement is hyperimmune.

We remark that weak truth-table reducibility is exactly the right kind of reducibility which gives non-trivial results in this context. This is because every nonzero c.e. degree contains a hypersimple set and every c.e. set is Turing reducible to a ranked set; but if $A \leqslant_{\mathrm{tt}} B$ and $B$ is ranked, then so is $A$.

Some connections between the notions are known:

Lemma 1.2 (Chisholm et al. [8]). Any initial segment of a scattered, computable linear ordering is ranked.

Lemma 1.3. If $A$ is c.e. and non-computable and is the $\omega$-part of a computable linear ordering of order-type $\omega+\omega^{*}$, then $A$ is hypersimple.

Proof. We note that if there is a computable function $f$ such that for all $n$,

$$
|(\omega \backslash A) \cap f(n)| \geqslant n,
$$

then we can enumerate $\omega \backslash A$ by listing, for all $n$, the $n$ rightmost points of $L \uparrow f(n)$.

Theorem 1.1 follows from the following two propositions, which in fact prove a little more.

Proposition 1.4. If $\mathbf{d}$ is a c.e. degree which is not totally $\omega$-c.e., then there is some c.e. set $B \leqslant_{T} \mathbf{d}$ which is not wtt-reducible to any hypersimple set. 
Proposition 1.5. If $\mathbf{d}$ is a c.e. degree which is totally $\omega$-c.e., then every $B \leqslant_{T} \mathbf{d}$ is wtt-reducible to a c.e. set which is the $\omega$-part of a computable linear ordering of order-type $\omega+\omega^{*}$.

These propositions are proved in section 2

Proof of Theorem 1.1. Let $\mathbf{d}>\mathbf{0}$ be a c.e. degree. If $\mathbf{d}$ is totally $\omega$-c.e., then by Proposition 1.5. every $C \in \mathbf{d}$ is wtt-reducible to a c.e. set $A$ which is a proper, non-empty initial segment of a computable linear ordering of order-type $\omega+\omega^{*}$. Of course $A$ is not computable, and so $A$ is both ranked and hypersimple.

If $\mathbf{d}$ is not totally $\omega$-c.e., then by Chisholm et al., there is some c.e. $A \in \mathbf{d}$ which is not wtt-reducible to any ranked set, and hence not to an initial segment of a scattered, computable linear ordering. By Proposition 1.4, there is some c.e. $B \leqslant_{T} \mathbf{d}$ which is not wtt-reducible to any hypersimple set; if $D \in \mathbf{d}$ is any c.e. set, then $B \oplus D$ is in $\mathbf{d}$ and again not wtt-reducible to any hypersimple set.

We mention that Proposition 1.4 does not simply follow from the result of Chisholm et al.; this is because there are hypersimple sets which are not wttreducible to any ranked sets. We prove that fact in section 3. On the other hand, Proposition 1.4 does not imply the result of Chisholm et al.; in the same section, we show that there is a ranked c.e. set which is not wtt-reducible to any hypersimple set.

In the second part of the paper, we turn to algorithmic randomness and in particular some natural notions of relative randomness. Recall that a Martin-Löf test is a method of coding all effective statistical tests. To wit, a computable collection of c.e. open sets $T=\left\{U_{n}: n \in \omega\right\}$ is a Martin-Löf test iff the Lebesgue measure $\mu\left(U_{n}\right)$ of $U_{n}$ is bounded by $2^{-n}$ for all $n$. Then a real $A$ passes the test $T$ iff $A \notin \bigcap_{n} U_{n}$. We say that a real is Martin-Löf random iff it passes all Martin-Löf tests 3 A universal Martin-Löf test $\left(V_{n}\right)$ is a Martin-Löf test with the property that $\bigcap_{n} V_{n}$ contains (and so, coincides with the set of) all non-random reals. The existence of a universal Martin-Löf test was an early result of Martin-Löf (see e.g. [32]). Martin-Löf randomness is a natural and robust notion of randomness in that it coincides with other methods of defining algorithmic randomness. For example a real should be random iff all its initial segments are incompressible, so that (like white noise) they have no patterns allowing compression. This intuition is proven correct by Schnorr's theorem, which says that $A$ is Martin-Löf random if and only if for all $n, K(A\lceil n)=n+O(1)$, where $A\lceil n$ denotes the first $n$ bits of $A$ and $K$ denotes prefix free Kolmogorov complexity 4

Schnorr's theorem suggests a natural method of calibrating randomness of reals: $A \leqslant_{K} B$ iff for all $n, K(A\lceil n) \leqslant K(B \nmid n)+O(1)$. Many measures of relative randomness implying this measure have been analysed. Of interest to us here is

\footnotetext{
${ }^{3}$ In the following, we sometimes say 'random' meaning 'Martin-Löf random'.

${ }^{4}$ Prefix-free Kolmogorov complexity is a modification of plain complexity $C$ which captures the intentional meaning of Kolmogorov complexity. Recall that for a machine $M$ the Kolmogorov complexity $C_{M}(\sigma)$ of a string $\sigma$ is the length of the shortest $\tau$ with $M(\tau)=\sigma$. Up to a constant, there is a universal machine $U$ with $C_{U}(\sigma) \leq C_{M}(\sigma)+O(1)$, for all $\sigma$, allowing us to define the (plain) Kolmogorov complexity. Prefix-free complexity solves certain deficiencies in this definition and simply asks that the domains of the machines have the property that if $U(\tau) \downarrow$, then for all strings $\rho$ comparable with $\tau, U(\rho) \uparrow$. We refer the reader to Li and Vitányi [30], Downey and Hirschfeldt [16] for more details.
} 
one inspired by strong reducibilities. In [17, 18] a strengthening of weak truthtable reducibility, namely computations where the use on the oracle on argument $n$ is $n+c$ for some constant $c$, was suggested as a possible measure of relative randomness. This reducibility has appeared in the literature with various names, e.g. strong weak truth table [17, 18, computable Lipschitz (due to a characterization of it in terms of effective Lipschitz functions) [4] and linear [6]. We will adopt the terminology in 4 and note it as $\leqslant_{\mathrm{cl}}$. It is nearly obvious that $\leqslant_{\mathrm{cl}}$ is a measure of relative randomness since a programme computing $n+c$ bits of $B$ will compute $n$ bits of $A$.

In 35, Yu and Ding proved that there are two left-c.e. reals (i.e. limits of computable increasing sequences of rationals) which had no common upper bound in the cl-degrees. In [7, Barmpalias and Lewis showed that there is a left-c.e. real which is not cl-reducible to any Martin-Löf random left-c.e. real.

The Yu-Ding Theorem and the Barmpalias-Lewis Theorem should be viewed in the context of the Kučera-Slaman Theorem [28], which shows that all random left-c.e. reals have the same Solovay degree (which is the greatest Solovay degree of left-c.e. reals). Here $\alpha$ is Solovay reducible to $\beta$ iff there is a constant $c$ and a partial computable $\varphi$ such that for all rationals $q<\beta, \varphi(q) \downarrow$ and $c|\beta-q|>$ $\alpha-\varphi(q)$. Thus, the Kučera-Slaman Theorem says that a computable sequence of rationals converging to some version of $\Omega$ can be efficiently converted into a sequence converging to any other version of $\Omega$ at more or less the same rate. The Yu-DingBarmpalias-Lewis material shows that there is no way to efficiently convert the bits of one version of $\Omega$ to some arbitrary left-c.e. real.

Both the Yu-Ding theorem and the Barmpalias-Lewis theorem characterise array computability.

Theorem 1.6. The following are equivalent for a c.e. degree $\mathbf{d}$ :

(1) There are left-c.e. reals $\alpha_{0}, \alpha_{1} \in \mathbf{d}$ which have no common upper bound in the cl-degrees of left-c.e. reals.

(2) There is a left-c.e. real $\alpha \in \mathbf{d}$ which is not cl-reducible to any random left-c.e. real.

(3) There is a set $A \in \mathbf{d}$ which is not cl-reducible to any random left-c.e. real.

(4) $\mathbf{d}$ is array non-computable.

To make the technical details of the proofs slightly simpler, we often work with an even more restrictive reducibility than computable Lipschitz: identity bounded Turing reducibility (ibT or $\leqslant_{\mathrm{ibT}}$ for short) is a computable Lipschitz reduction for which the constant $c$ is 0 . This reducibility was introduced by Soare in connection with applications of computability theory to differential geometry (see [10, 5]). Even though the degree structures are different, for our purposes, we may work with either:

\section{Lemma 1.7.}

(1) Left-c.e. reals $\alpha_{0}$ and $\alpha_{1}$ have a common upper bound in the cl-degrees of left-c.e. reals iff they have a common upper bound in the ibT-degrees of the left-c.e. reals.

(2) $A$ set $A$ is cl-reducible to a random left-c.e. real iff it is ibT-reducible to a random left-c.e. real.

Proof. If $\alpha \leqslant_{\mathrm{cl}} \beta$ with constant $c$, then $\alpha \leqslant_{\mathrm{ibT}} \beta \uparrow[c, \infty)$. If $\beta$ is random, then so is $\beta \uparrow[c, \infty)$. 
Theorem 1.6 follows from the following four propositions, which will be proved in section 4, Again we prove slightly more.

Proposition 1.8. If $\mathbf{d}$ is c.e. and array non-computable, then there are left-c.e. reals $\alpha_{0}, \alpha_{1} \in \mathbf{d}$ which have no common upper bound in the ibT degrees of left-c.e. reals.

For the proof of this proposition, we give a significantly simplified account of the verifications of the construction of Yu and Ding, which appeared in [35] and [4.

Proposition 1.9. If $A_{0}$ and $A_{1}$ are sets, both of which have c.e., array computable degree, then there is a left-c.e. real $\beta$ such that $A_{0}, A_{1} \leqslant \mathrm{ibT} \beta$.

The fact that we don't need $A_{0} \oplus A_{1}$ to have array computable degree may be somehow related to the fact [19] that the wtt degrees of c.e. sets which do not contain array non-computable sets are closed under join.

Proposition 1.10. If $\mathbf{d}$ is c.e. and array non-computable, then there is some leftc.e. real $\alpha \in \mathbf{d}$ which is not ibT-reducible to any random left-c.e. real.

Here we give a construction which is significantly simpler than that which appeared in [7].

Proposition 1.11. If $\mathbf{d}$ is c.e. and array computable, and $A \leqslant_{T} \mathbf{d}$, then $A$ is ibT-reducible to some random left-c.e. real.

To clarify the situation regarding the cl-degrees of random left-c.e. reals, Frank Stephan has proved the following fact:

Theorem 1.12. There are two random left-c.e. reals which are not cl-equivalent.

We give Stephan's proof in section 6 .

Finally, we somewhat extend Proposition 1.10 beyond the c.e. degrees. We remark that Hirschfeldt (see [16]) has constructed a real (not left-c.e.) which is not ibT-reducible to any random real, indeed to any complex real. Recall that a set $A$ is called complex (by Kjos-Hanssen, Merkle and Stephan, [25]) if there is a nondecreasing unbounded computable function $f$ such that $K(A\lceil n)>f(n)$ for all $n$; thus this is a weakening of the notion of randomness. Using Hirschfeldt's technique and sufficient genericity which is available from non- $\mathrm{GL}_{2}$ permitting (recall that a degree $\mathbf{d}$ is called generalised low if $_{2} \mathbf{d}^{\prime \prime} \leqslant\left(\mathbf{d} \vee \mathbf{0}^{\prime}\right)^{\prime}$; equivalently, every $f \leqslant_{T}\left(\mathbf{d} \vee \mathbf{0}^{\prime}\right)$ is not dominated by some $g \leqslant_{T} \mathbf{d}$ ), we prove the following in section 5 ;

Theorem 1.13. If $\mathbf{d}$ is not generalised low $w_{2}$, then there is some $A \leqslant_{T} \mathbf{d}$ which is not ibT-reducible to any complex real.

This result complements a line of recent knowledge about using strong reducibilities with random oracles. Early on, Gács [23] and Kučera [26, 27] showed that every real is wtt-reducible to a random one. In fact, Gács proved that the use of the reduction can be $n+o(n)$, that is, asymptotically close to an ibT reduction (see 31]). Recently, Doty [11] showed that for every $A \in 2^{\omega}$ there is some random $R \geqslant_{T} A$ such that the limit superior of the use of computing $A\lceil n$ from $R$, divided by $n$, is exactly the effective packing dimension $\operatorname{Dim}(A)$ of $A$. Thus if $\operatorname{Dim}(A)<1$, then there is some random $R \geqslant_{\mathrm{ibT}} A$. For example, if $\mathbf{d}$ is c.e. traceable 5 then every

\footnotetext{
${ }^{5}$ I.e. there is an order function $h$ such that every function $f$ which is computable from $\mathbf{d}$ has a c.e. trace with bound $h$. A c.e. trace with bound $h$ is a uniformly c.e. sequence of sets $\left\langle T_{n}\right\rangle$ such that $\left|T_{n}\right| \leq h(n)$ for all $n$. We say that $\left\langle T_{n}\right\rangle$ is a trace for $f$ if $f(n) \in T_{n}$ for each $n$.
} 
$A \in \mathbf{d}$ has effective packing dimension zero [14] and so every $A \in \mathbf{d}$ is ibT-reducible to a random, hence to a complex, real. The space between the c.e. traceable degrees and the non- $\mathrm{GL}_{2}$ degrees remains a mystery. So we ask:

Question 1.14. Which Turing degrees contain sets which are not ibT-reducible to random (or complex) reals?

The first step, we suspect, would be to analyse the array non-computable degrees.

\section{WEAK TRUTh-TABLE REDUCtions AND TOtALLY $\omega$-C.E. DEGREES}

2.1. Non-totally $\boldsymbol{\omega}$-c.e. permitting. Here we prove Proposition 1.4 if $\mathbf{d}$ is c.e. and not totally $\omega$-c.e., then there is some c.e. $A \leqslant_{T} \mathbf{d}$ which is not weak-truth-table reducible to any hypersimple set. To do so, we apply non-totally $\omega$-c.e. permitting to the construction of a c.e. set which is not weak-truth-table reducible to any hypersimple set. To make the argument clear, we first review that construction, which appeared in [2, 3].

Let $\left\langle\Phi_{e}\right\rangle$ be an effective enumeration of all truth-table functionals: $\Phi_{e}$ is a Turing functional whose use function is bounded by the computable function $\varphi_{e}$ (of course, some of the use functions may be partial, in which case we assume the corresponding computations do not converge). In enumerating the desired set $A$, the requirements we need to meet are

$$
R_{e, i}: \text { If } \Phi_{e}^{W_{i}}=A \text {, then } W_{i} \text { is not hypersimple. }
$$

The strategy for a single requirement $R_{e, i}$ is the following. We inductively define sets of followers $X_{0}, X_{1}, \ldots$ such that $\max X_{n}<\min X_{n+1}$ which will be used for diagonalisation against $\Phi_{e}^{W_{i}}=A$. If the wtt reduction $\Phi_{e}^{W_{i}}$ is total, then we can compute an increasing sequence $u_{0}<u_{1}<u_{2}<\cdots$ such that for all $n$, for all $x \in X_{n}$, the use of the computation $\Phi_{e}^{W_{i}}(x)$ is smaller than $u_{n}$. The key is that the size of the sets of followers $X_{n}$ grows fast: we ensure that $\left|X_{n}\right|>u_{n-1}$. Then if $W_{i}$ is indeed hypersimple, then at some stage of the construction we will discover some $n$ such that the entire interval $\left[u_{n-1}, u_{n}\right)$ is a subset of $W_{i}$. The size of $X_{n}$ then allows us to keep diagonalising against any future configuration of $W_{i} \uparrow u_{n}$, since there are at most $u_{n}$ of those.

The sets of followers for different requirements can be kept disjoint, and so there is no interaction between distinct requirements, which act completely independently. Thus we have no need for the priority mechanism.

We now add the permitting component and describe the proof. It is quite straightforward. Say we are given a c.e. set $D$ and some $g=\Gamma(D)$ which has no $\omega$-c.e. approximation. Each requirement proceeds as before, setting up sets of followers $X_{n}$ and computing the sequence of $u_{n}$ 's. When we want to start enumerating followers from some $X_{n}$ into $A$ (upon discovery that $\left[u_{n-1}, u_{n}\right) \subseteq W_{i}$ ) we know that we need at most $\left|X_{n}\right|$ many permissions from $D$ to successfully meet the requirement. Now we know that if $W_{i}$ is hypersimple, then in fact there are infinitely many $n$ such that $\left[u_{n-1}, u_{n}\right) \subseteq W_{i}$. So if we tie the $D$-use for permitting $x \in X_{n}$ to the $D$-use of computing $g\left\lceil a_{n}\right.$ (via $\Gamma$ ), then failure to obtain permission on each of these $n$ 's will yield an $\omega$-c.e. approximation for $g$.

Construction. We now give the formal details. We give the instructions for a single requirement $R_{e, i}$, because again, there is no interaction between distinct requirements, even though they implicitly collaborate in building the reduction from $A$ to 
$D$. Let, for $a<\omega, \gamma(a)$ (at a stage $s$ ) be the current use of computing $g\lceil a$ via $\Gamma(D)$. [Whenever the construction asks for a value $\gamma(a)$ we run the enumeration of $D$ forward until we get $|\operatorname{dom} \Gamma(D)|>a$.]

Setting up $X_{n}$ :

Let $X_{n}$ consist of $u_{n-1}+1$ large numbers which have not been used before. Also choose a large number $a_{n}$.

Attacking with $X_{n}$ at stage s:

Pick any $x \in X_{n} \backslash A$ and let $v=\gamma\left(a_{n}\right)$ (at stage $s$ ). Wait for the least stage $t>s$ such that $D_{s}\left\lceil v \neq D_{t} \uparrow v\right.$. If such a stage appears, enumerate $x$ into $A$ at that stage.

The instructions for $R_{e, i}$ consist of two parts, which are performed in parallel.

(1) Let $u_{-1}=0$. Set up $X_{0}$. After setting up $X_{n}$, wait for a stage at which $\Phi_{e}^{W_{i}}$ agrees with $A \uparrow \max X_{n}+1$. Let $u_{n}=\varphi_{e}\left(\max X_{n}+1\right)$ be the use of that computation, and use that value to set up $X_{n+1}$.

(2) For any $n$, if $\left[u_{n-1}, u_{n}\right) \subseteq W_{i}$ and $\Phi_{e}^{W_{i}}$ agrees with $A \uparrow \max X_{n}+1$ (and we are not currently attacking with $X_{n}$ ), then attack with $X_{n}$.

Again note that setting up new $X_{n}$ 's proceeds while possible attacks are made with older $X_{m}$ 's. Also, several attacks (with distinct $X_{m}$ 's) may be performed simultaneously.

\section{Verification.}

Lemma 2.1. $A \leqslant_{T} D$.

Proof. Let $x<\omega$. To find whether $x \in A$, first go to stage $x$ and see if there is some requirement $R_{e, i}$ which has chosen $x$ to be an element of a follower set $X_{n}$ belonging to this requirement. If not, then $x \notin A$. Otherwise, let $a_{n}$ be the parameter chosen when $X_{n}$ was set up. Wait for a stage $s$ at which $g\left\lceil a_{n} \subset \Gamma(D)\right.$ via a $D$-correct computation. Then $x \in A$ iff $x \in A_{s}$.

Lemma 2.2. $A$ is not wtt-reducible to any hypersimple set.

Proof. Suppose for a contradiction that $W_{i}$ is hypersimple and $\Phi_{e}^{W_{i}}=A$ (with computable bound $\varphi_{e}$ on the use). Since $\Phi_{e}^{W_{i}}$ is total, the requirement $R_{e, i}$ sets up follower sets $X_{n}$ for every $n<\omega$ and computes the associated sequence $\left\langle u_{n}\right\rangle$. By assumption, the set

$$
B=\left\{n<\omega:\left[u_{n-1}, u_{n}\right) \subset W_{i}\right\}
$$

is infinite. For each $n \in B$, an attack with $X_{n}$ commences at some stage. Since $\Phi_{e}^{W_{i}}$ computes $A$ correctly, for no $n \in B$ do we get to enumerate all of $X_{n}$ into $A$. We cannot get stuck waiting for another agreement between $\Phi_{e}^{W_{i}}$ and $A\left\lceil\max X_{n}+1\right.$, so it must be that for each $n \in B$, an attack with $X_{n}$ gets stuck waiting for permission from $D$.

This means that if we approximate, for $n \in B, g\left\lceil a_{n}\right.$ to be its value at a stage at which an attack with $X_{n}$ is started, then for each $n \in B$ there are at most $\left|X_{n}\right|$ many mind-changes about $g\left\lceil a_{n}\right.$. Since the sequence $\left\langle a_{n}\right\rangle_{n \in B}$ is computable, this gives us an $\omega$-c.e. approximation for $g$, and a contradiction. 
2.2. Totally $\omega$-c.e. anti-permitting. We prove Proposition 1.5 under the simplifying hypothesis that the set $B$ is c.e. Since the totally $\omega$-c.e. degrees are downward closed, we simply let $B=D$ be a c.e. set of totally $\omega$-c.e. degree, and show that there is some computable linear ordering $L$ of order-type $\omega+\omega^{*}$ such that the $\omega$-part of $L$ is c.e. and wtt-computes $D$. The reader may find it interesting to note how the proof behaves like a mirror image of the proof of the previous section.

The proof follows the style of the "anti-permitting" arguments of [15, 12, 13]. The general idea is that we globally construct some function $\Gamma(D)$. If we knew some $\omega$-c.e. approximation to $\Gamma(D)$, then we could use that approximation to limit changes in $D$ (as we would not be able to change $\Gamma(D)$ too many times). In the present case we would use this to correctly compute $D$ from a set $A$ we are building. There is a small inconvenience in that although we know that $\Gamma(D)$ is $\omega$-c.e., we cannot effectively get an $\omega$-c.e. approximation to $\Gamma(D)$. That is why we opt for a non-uniform construction: for every guess at such an approximation, we build a separate set $A$ and a reduction from $D$ to $A$. The correct guess will build a successful reduction and a set with the desired property (here, being the $\omega$-part of a computable linear ordering of type $\left.\omega+\omega^{*}\right)$. The only interaction between the different guesses is that together they need to construct $\Gamma(D)$; thus even if a guess is incorrect, we need to ensure that on the inputs under this guess' responsibility, $\Gamma(D)$ is defined.

The heart of the proof is the strategy, given an approximation for $\Gamma(D)$. Suppose that we are given a correct approximation $g_{s}(x)$ to $\Gamma(D)$, where the value of $g_{s}(x)$ changes at most $h(x)$ many times (with $h$ computable). To begin, we choose a large number $a_{0}$, and set up $h\left(a_{0}\right)+2$ many points in the computable linear ordering $<_{L}$ that we are building. We call the collection of points we set up the first layer $L_{0}$ of $<_{L}$. To begin, we declare that $A$ (which will be the $\omega$-part of $<_{L}$ ) contains only the leftmost point of $L_{0}$. We define $\Gamma\left(D, a_{0}\right)$ with large use $\gamma\left(a_{0}\right)$. When we get confirmation that the current version of $D \uparrow \gamma\left(a_{0}\right)$ is correct by obtaining a stage $s$ at which $g_{s}\left(a_{0}\right)=\Gamma\left(D, a_{0}\right)$, then we declare that $A \uparrow L_{0}$ (that is, the information that from the points in $L_{0}$, only the leftmost one is in $A$ ) computes $D \uparrow \gamma\left(a_{0}\right)$. [If $D$ changes before we get such a confirmation, then we start again with the new value of $D \uparrow \gamma\left(a_{0}\right)$. Note that if $g_{s}$ is not a correct approximation for $\Gamma(D)$, then $D\left\lceil\gamma\left(a_{0}\right)\right.$ could be correct, yet we would never receive confirmation for this fact. The construction for this incorrect guess at an approximation is then stuck. But note that $\Gamma\left(D, a_{0}\right)$ is nevertheless well defined and so this eventuality doesn't spoil the totality of $\Gamma(D)$.]

We then proceed to choose the next "unpermitting number" $a_{1}$ and lay the next layer $L_{1}$ of $h\left(a_{1}\right)+2$ points of $<_{L}$ which will be put in the cut between $A$ and its complement, namely, between the leftmost point of $L_{0}$ and the next point of $L_{0}$. We now repeat the process by enumerating the leftmost point of $L_{1}$ into $A$, defining $\Gamma\left(D, a_{1}\right)$ with large use $\gamma\left(a_{1}\right)$ and upon confirmation by $g_{s}\left(a_{1}\right)$, we stipulate that $A \uparrow\left(L_{0} \cup L_{1}\right)$ computes $D\left\lceil\gamma\left(a_{1}\right)\right.$.

Of course, the main issue is what happens if $D$ does change, making the reduction to $A$ incorrect. We then need to change $A$ to react to the $D$-change. Suppose, for example, that $D \uparrow \gamma\left(a_{0}\right)$ changed from its original value. We then decide to enumerate the second leftmost point of $L_{0}$ into $A$. This means that all the subsequent layers we put down have to be abandoned (and enumerated into $A$ ). This does not affect the order-type of $<_{L}$ because it means just adding finitely many 
points to the $\omega$-part $A$. But it does invalidate all the computations we commited to, of $D\left\lceil\gamma\left(a_{0}\right)\right.$ from $A\left\lceil L_{0}, D\left\lceil\gamma\left(a_{1}\right)\right.\right.$ from $A\left\lceil\left(L_{0} \cup L_{1}\right)\right.$, etc., and allows us to define new ones. The fact that we only make such commitments when a confirmation of $D$ is obtained, and the fact that $g_{s}$ is indeed an $h$-c.e. approximation for $\Gamma(D)$, tells us that the process will halt before we run out of points in the layer.

There is one last delicate matter we need to address. We need to build a weak truth-table reduction of $D$ to $A$. When we set up a layer such as $L_{1}$, we commit to using only $L_{0} \cup L_{1}$ for computing $D \uparrow \gamma\left(a_{1}\right)$. If later we abandon that version of $L_{1}$, we cannot lift the use to compute $D\left\lceil\gamma\left(a_{1}\right)\right.$ from the new $L_{1}$. The only thing that we can do is require that $D\left\lceil\gamma\left(a_{1}\right)\right.$ (for the old value of $\gamma\left(a_{1}\right)$ ) be computable by $A \uparrow L_{0}$, because we enumerated the old $L_{1}$ in its entirety into $A$. How can we ensure that $D\left\lceil\gamma\left(a_{1}\right)\right.$ doesn't change more than $h\left(a_{0}\right)$ many times? This we do by updating the value of $\gamma\left(a_{0}\right)$ to be larger than the old $\gamma\left(a_{1}\right)$, and we can do this exactly because $D \uparrow \gamma\left(a_{0}\right)$ has just changed. We note that this is necessary: without this feature, we wouldn't need to ever change $\gamma\left(a_{n}\right)$, and then we'd have $\Gamma(D) \leqslant$ wtt $D$ and we'd be able to prove the lemma for all c.e. sets $D$, which is of course false.

Construction. Let $\left\langle g_{e}, h_{e}\right\rangle$ be an effective enumeration of all pairs of partial $\omega$ c.e. approximations: so $g_{e}(x, s)$ is a total computable function, $h_{e}$ is a partial computable function, and for each $x$, the number of $s$ such that $g_{e}(x, s) \neq g_{e}(x, s+1)$ is bounded by $h_{e}(x)$ (and is 0 if $h_{e}(x) \uparrow$ ). We are given a c.e. set $D$; we define a Turing functional $\Gamma$. For each $e$ we also define a computable linear ordering $<_{e}$ and other auxiliary sets. As discussed, apart from building $\Gamma$, the construction for each $e$ is completely independent, so we describe the instructions for $e$ and drop the subscript $e$ everywhere. So fix $e$, and let $g_{s}(x)=g_{e}(x, s)$ and $h(x)=h_{e}(x)$. We build the linear ordering $<_{L}=<_{e}$ and approximate finite sets $L_{n}=L_{e, n}$ and enumerate a set $A=A_{e}$.

The main module for $e$ is:

(1) Let $n$ be the least integer such that $L_{n}$ is currently undefined. Let $a_{n}$ be the least number not picked as an unpermitting number by any $e^{\prime}$ (including $e$ itself). Define $\Gamma\left(D, a_{n}\right)$ to be the stage number $s$ with use $\gamma\left(a_{n}\right)=s$. Wait for $h\left(a_{n}\right)$ to converge and for $g_{t}\left(a_{n}\right)=s$. If, while waiting, $D\lceil s$ changes, redefine $\Gamma\left(D, a_{n}\right)=s$ with use $s$ (note, this is the original $s$, not the new stage number).

(2) Let $L_{n}$ be a collection of $h\left(a_{n}\right)+2$ many points not currently in the domain of $<_{L}$. Extend the definition of $<_{L}$ to linearly order $L_{n}$ (say to agree with the natural ordering on $\mathbb{N}$ ) and place $L_{n}$ between $\max _{<L} A$ and its successor in $<_{L}$. Enumerate $\min L_{n}$ into $A$. Return to (1).

There is one interrupt procedure for $e$. The $n$-interrupt condition at a stage $t$ is: $s<t$ is the last stage before $t$ at which $L_{n}$ is defined (step (2) of the main module) or a single number is enumerated into $A \cap L_{n}$ (step (2) of an $n$-interrupt), $L_{n}$ is not cancelled between stages $s$ and $t$, and

$$
D \uparrow \gamma\left(a_{n}\right)[s] \neq D \uparrow \gamma\left(a_{n}\right)[t]
$$

The instructions for the $n$-interrupt are:

(1) For all $n^{\prime}>n$, enumerate $L_{n^{\prime}}$ into $A$ and declare $L_{n^{\prime}}$ undefined. Also, set $\Gamma\left(D, a_{n^{\prime}}\right)=0$ with use 0 and declare $a_{n^{\prime}}$ undefined. Redefine $\Gamma\left(D, a_{n}\right)=t$ 
with new use $\gamma\left(a_{n}\right)=t$. Wait for $g_{r}\left(a_{n}\right)=t$. In the meanwhile, if $D \nmid t$ changes, redefine $\Gamma\left(D, a_{n}\right)=t$ with use $t$.

(2) Enumerate $\min \left(L_{n} \backslash A\right)$ into $A$. Return to the main module.

Verification. First, we note that the instructions are consistent, namely, that step (2) of the interrupt process can always be carried out.

Lemma 2.3. For every e, at every stage $s$, for every $n$ such that $L_{n}=L_{n, e}$ is defined, $L_{n} \nsubseteq A$ (where $A=A_{e}$ ).

Proof. As long as $L_{n}$ is not cancelled, the size of $A \cap L_{n}$ is $1+$ the number of times (since the current version of $L_{n}$ was set up) an $n$-interrupt arrived at step (2). For each such step we have a new guess for the value of $g_{s}\left(a_{n}\right)$ and so

$$
\left|A \cap L_{n}\right| \leqslant 1+h\left(a_{n}\right)<\left|L_{n}\right| .
$$

Lemma 2.4. $\Gamma(D)$ is total.

Proof. Let $a<\omega$. By induction assume that every $b<a$ is in the domain of $\Gamma(D)$. Then there is some $e$, some stage $s$ and some $n$ such that $e$ picks $a$ as $a_{n}$ at stage $s$. If $e$ ever cancels $L_{n}$, then we define $\Gamma(D, a)$ with use 0 and so $\Gamma(D, n) \downarrow$. If $e$ ever gets stuck at step (1) of the main module (when setting up $L_{n}$ ) or at step (1) of an $n$-interrupt, then we keep defining $\Gamma(D, a)=t$ with use $t$ for some stage $t$; eventually, $D\lceil t$ stabilizes and so this definition becomes permanent.

Otherwise, we first define $\Gamma(D, a)=s$ with use $s$ when setting up $L_{n}$, and at every $n$-interrupt after that, we redefine $\Gamma(D, a)=t$ with use $t$ for some $t$. As we've seen before, this happens finitely many times. After each time, if $D \uparrow t$ changes, then we call an $n$-interrupt again. Thus eventually one of these definitions is permanent.

By our assumption that $\operatorname{deg}_{T}(D)$ is totally $\omega$-c.e., we can fix an $e$ such that $\left(g_{e}, h_{e}\right)$ is an $\omega$-c.e. approximation for $\Gamma(D)$. From now on, drop all subscripts $e$. Note that neither the main module for $e$ nor any interrupt for $e$ ever gets stuck waiting at step (1).

Lemma 2.5. $<_{L}$ is a computable linear ordering of order-type $\omega+\omega^{*}$, and $A$ is the $\omega$-part of $<_{L}$.

Proof. By induction on $n$ we see that for every $n$, there is some version of $L_{n}$ which is never cancelled. Suppose that a version of $L_{n-1}$ is set up at stage $s$ and is not later cancelled. Then there is a stage $t \geqslant s$ at which the last element of $A \cap L_{n-1}$ gets enumerated into $A$. Immediately after that stage, a new $L_{n}$ is set up. This $L_{n}$ will never be cancelled, because an $n-1$ interrupt does not get stuck at step (1) and so necessarily enumerates another point into $A \cap L_{n-1}$.

For every version of $L_{n}$, including the final one, $A \cap L_{n}$ is non-empty, and so $A$ is infinite. On the other hand, if a point $x$ is enumerated into $A$ at stage $s$, then no points are later placed in $<_{L}$ to the left of $x$, so the initial segment of $<_{L}$ determined by $x$ is finite. Thus the order type of $A$ is $\omega$. It is also easy to see that the instructions ensure that $A$ is an initial segment of $<_{L}$.

For every permanent version of any $L_{n}, \max L_{n}$ never gets enumerated into $A$ and so the complement of $A$ is infinite. In fact, the complement of $A$ is the union of $L_{n} \backslash A$ for all permanent $L_{n}$ 's. For each point in that complement, after it is added to $<_{L}$ (when the permanent $L_{n}$ is set up), no new points to the right are ever added. Thus the order-type of the complement of $A$ is $\omega^{*}$. 
Lemma 2.6. $D \leqslant_{\mathrm{wtt}} A$.

Proof. Suppose that at stage $s$, a (not necessarily permanent) $L_{n}$ is set up and $a_{n}$ is chosen. Then $s=\gamma_{s}\left(a_{n}\right)$. We show how to compute $D\left\lceil s\right.$ from $A\left\lceil\bigcup_{m \leqslant n} L_{m}\right.$, where the $L_{m}$ 's are their versions at stage $s$.

Let $m \leqslant n$ be the greatest integer such that after stage $s, L_{m}$ is never cancelled (such an $m$ exists, because $L_{0}$ is never cancelled, and can be calculated from $A \uparrow$ $\bigcup_{m \leqslant n} L_{m}$ by looking at the last element of each $L_{m}$ ). Let $t$ be the last stage at which we go through step (2) of the main module (setting up $L_{m}$ ) or an $m$-interrupt, and let $r<t$ be the stage at which the corresponding step (1) is started. $A\left\lceil L_{m}\right.$ can calculate these stages. The main point is that $r=\gamma_{r}\left(a_{m}\right)=\gamma\left(a_{m}\right)$ is not smaller than $s$. For if $L_{n}$ is never cancelled, then $m=n$ and then $r \geqslant s$; or $m<n$, in which case $L_{n}$ is cancelled at stage $r$ and so $r>s$.

Since there is no $m$-interrupt after stage $t$, we know that $D \uparrow r=D_{t} \uparrow r$. So $D \uparrow s$ doesn't change after stage $t$.

2.3. What if $B$ is not c.e.? We now explain how to change the proof above in the case that the given set $B \leqslant_{T} \mathbf{d}$ is not c.e., and thus indicate how to prove the full version of Proposition 1.5. Let $D \in \mathbf{d}$ be c.e., and let $\Theta$ be a Turing functional such that $\Theta(D)=B$. The construction now is almost identical to the construction above, building $\Gamma(D)$, etc., except that we tie the use of $\Theta$ computations to that of $\Gamma$ computations. Namely, we require that if $a_{n}$ is chosen as an unpermitting number for some guess $\left(g_{e}, h_{e}\right)$, then at all stages $s$, the use $\gamma_{s}\left(a_{n}\right)$ is greater than the use of computing $\Theta(D)\lceil s$ at that stage. [Because $\Theta(D)$ is total, by speeding up the enumeration of $D$, we may assume that for all $s, \Theta(D)\lceil s \downarrow$ at stage $s$.] So instead of computing $D\left\lceil s, A\left\lceil\bigcup_{m \leqslant n} L_{m}\right.\right.$ is responsible for computing $\Theta(D) \uparrow s$. Before confirmation, if $D$ changes below this $\gamma\left(a_{n}\right)$, then we may need to update $\gamma\left(a_{n}\right)$ because of an increase in the use of $\theta(s)$. But this doesn't spin out of control because $\Theta(D)$ is total, so $\theta(s)$ reaches a limit.

When $D$ changes after confirmation, and an interrupt is called, then we need to shift responsibilities as above: some smaller $m$ is handed with the burden of computing $\Theta(D) \uparrow s$. The general geometry of the construction is not changed much.

Construction. Again we are given $\left\langle g_{e}, h_{e}\right\rangle$ and $D$, and this time, also a Turing functional $\Theta$ such that $\Theta(D)$ is total. We define a Turing functional $\Gamma$, and computable linear orderings $<_{e}$, and other auxiliary sets. Apart from the use markers $\gamma_{s}(a)$, we also need to define placement markers $\delta(n)=\delta_{e}(n)$; the idea is that $A\left\lceil\bigcup_{m \leqslant n} L_{m}\right.$ computes $\Theta(D) \uparrow \delta(n)$.

The main module for $e$ is:

(1) Let $n$ be the least integer such that $L_{n}$ is currently undefined. Let $a_{n}$ be the least number not picked as an unpermitting number by any $e^{\prime}$ (including $e$ itself). Define $\delta(n)=s$ (the current stage number), and define $\Gamma\left(D, a_{n}\right)=s$ with use $\theta_{s}(\delta(n))$. Wait for $g_{t}\left(a_{n}\right)=s$.

If, while waiting, $D\left\lceil\gamma_{s}\left(a_{n}\right)\right.$ changes, redefine $\Gamma\left(D, a_{n}\right)=s$ with use $\theta_{t}(\delta(n))$ (for the current stage $\left.t\right)$.

(2) Let $L_{n}$ be a collection of $h\left(a_{n}\right)+2$ many points not currently in the domain of $<_{L}$. Extend the definition of $<_{L}$ to linearly order $L_{n}$ (say to agree with the natural ordering on $\mathbb{N}$ ) and place $L_{n}$ between $\max _{<L} A$ and its successor in $<_{L}$. Enumerate $\min L_{n}$ into $A$. Return to (1). 
The $n$-interrupt condition for $e$ at a stage $t$ is:

$s<t$ is the last stage before $t$ at which $L_{n}$ is defined (step (2) of the main module) or a single number is enumerated into $A \cap L_{n}$ (step (2) of an $n$-interrupt), $L_{n}$ is not cancelled between stages $s$ and $t$, and

$$
D \uparrow \gamma\left(a_{n}\right)[s] \neq D \uparrow \gamma\left(a_{n}\right)[t]
$$

The instructions for the $n$-interrupt are:

(1) For all $n^{\prime}>n$, enumerate $L_{n^{\prime}}$ into $A$ and declare $L_{n^{\prime}}$ undefined. Also, set $\Gamma\left(D, a_{n^{\prime}}\right)=0$ with use 0 and declare $a_{n^{\prime}}$ and $\delta\left(n^{\prime}\right)$ undefined.

Redefine $\delta(n)=t$ and $\Gamma\left(D, a_{n}\right)=t$ with new use $\gamma\left(a_{n}\right)=\theta_{t}(\delta(n))$. Wait for $g_{r}\left(a_{n}\right)=t$. In the meanwhile, if $D \uparrow t$ changes, redefine $\Gamma\left(D, a_{n}\right)=t$ with use the current $\theta(\delta(n))$.

(2) Enumerate $\min \left(L_{n} \backslash A\right)$ into $A$. Return to the main module.

Verification. Again, the instructions are consistent:

Lemma 2.7. For every e, at every stage $s$, for every $n$ such that $L_{n}=L_{n, e}$ is defined, $L_{n} \nsubseteq A$ (where $A=A_{e}$ ).

The proof is identical to that of Lemma 2.3 .

Lemma 2.8. $\Gamma(D)$ is total.

Proof. Identical to the proof of Lemma 2.4. except that if the guess is wrong and we get stuck, after defining $\delta(n)=s$ and $\Gamma\left(D, a_{n}\right)=s$ with use $\theta(\delta(n))$, waiting for $g_{t}\left(a_{n}\right)=s$, then $\delta(n)$ (for this $e$ ) is not redefined after stage $s$, and so $\theta(\delta(n)$ ) eventually reaches a limit $u$; after that, we keep redefining $\Gamma\left(D, a_{n}\right)=s$ with use $u$, and this process halts once $D \uparrow u$ stabilizes.

Again we pick a correct guess $(g, h)$ and get that the ordering built for the correct guess is of type $\omega+\omega^{*}$, with $A$ being the $\omega$-part.

Lemma 2.9. $\Theta(D) \leqslant$ wtt $A$.

Proof. Suppose that at stage $s$, a (not necessarily permanent) $L_{n}$ is set up and $a_{n}$ is chosen. Then $s=\delta_{s}(n)$. We show how to compute $\Theta(D) \uparrow s$ from $A \uparrow \bigcup_{m \leqslant n} L_{m}$, where the $L_{m}$ 's are their versions at stage $s$.

Let $m \leqslant n$ be the greatest integer such that after stage $s, L_{m}$ is never cancelled. Let $t$ be the last stage at which we go through step (2) of the main module (setting up $\left.L_{m}\right)$ or an $m$-interrupt, and let $r<t$ be the stage at which the corresponding step (1) is started. Then $r=\delta(n)$ (as is set at stage $r$ and is not altered later) is not smaller than $s$; the proof is the same as in Lemma 2.6 for $\gamma\left(a_{n}\right)$.

Let $u=\theta_{t}(r)$. Since there is no $m$-interrupt after stage $t$, we know that $D \uparrow u=$ $D_{t} \uparrow u$. So $\Theta(D) \uparrow s$ doesn't change after stage $t$.

\section{Hypersimplicity AND RANKED SETS IN THE WTT DEGREES}

3.1. A hypersimple set which is not wtt-reducible to a ranked set. Here we prove the following fact:

Proposition 3.1. There is a hypersimple set which is not wtt-reducible to any ranked set. 
To prove this proposition, we would like to closely follow the construction (from [8] ) of a c.e. set which is not wtt-reducible to any ranked set. If the set we enumerate is $A$, the diagonalisation requirements to meet are:

$R_{e}$ : If $X \in \mathcal{P}_{e}$ and $\Phi_{e}^{X}=A$, then $\mathcal{P}_{e}$ is uncountable.

Here $\left(\mathcal{P}_{e}, \Phi_{e}\right)$ is a list of all pairs of $\Pi_{1}^{0}$ classes and wtt-functionals (as usual, $\varphi_{e}$ denotes a computable bound on the use of $\Phi_{e}$ ). The general plan from 8 is to devote a column of $\omega$ to each requirement, and then diagonalise on finitely many elements of the column against a finite clopen covering of $\mathcal{P}_{e}$ whose elements are small enough (that is, the strings are longer than the use of $\Phi_{e}$ ); a combinatorial lemma says that if $\mathcal{P}_{e}$ is countable, then such a covering must show up eventually.

In our case, we also want to make $A$ hypersimple. So we want to meet the following requirements:

$P_{e}$ : If $g_{e}$ is total and $\left\langle D_{g_{e}(n)}\right\rangle_{n<\omega}$ consists of pairwise disjoint sets, then there is some $n$ such that $D_{g_{e}(n)} \subset A$.

Here $\left(g_{e}\right)$ is an enumeration of all partial computable functions, and $D_{x}$ is an enumeration of all finite sets of natural numbers.

The hypersimplicity requirements do not interact well with the general plan; a requirement $R_{e}$ cannot keep a whole column to itself and expect even lower priority requirements to never enumerate elements of the column into $A$. It can, however, using finitely much restraint, ensure that infinitely much of $\omega$ is available for diagonalisation. Of course the part of $\omega$, namely $\omega \backslash A$, which is left for diagonalisation is not computable; this makes the combinatorial lemma useless. Using incompleteness, in particular Arslanov's criterion and DNR functions, we salvage enough of the lemma and push through the construction. Recall that a function $g$ is DNR (diagonally non-recursive) if $g(e) \neq \varphi_{e}(e)$ for all $e$ such that $\varphi_{e}(e)$ is defined.

Relativised complexity. We start with the combinatorial lemma. For any function $g: \omega \rightarrow \omega$, let $\mathcal{Q}_{g}$ be the collection of sets $X \in 2^{\omega}$ such that for all $n, C(X\lceil g(n)) \geqslant$ $n$. The class $\mathcal{Q}_{g}$ is a $\Pi_{1}^{0}(g)$-class.

Lemma 3.2. For all $X \in \mathcal{Q}_{g}, X \oplus g$ computes a DNR function.

Proof. This is implicit in 25. Proposition 6]; the function $n \mapsto X \uparrow g(n)$ differs from $\varphi_{n}(n)$ for almost all $n$ because the plain complexity of $\varphi_{n}(n)$ is at most $C(n)$, which is smaller than $n$.

The following is analogous to [8, Theorem 4.7].

Lemma 3.3. If $\mathcal{P}$ is a countable $\Pi_{1}^{0}$-class and $g$ does not compute a DNR function, then $\mathcal{P} \cap \mathcal{Q}_{g}=\emptyset$.

Proof. The point is that no $X \in \mathcal{Q}_{g}$ is computable in $g$, and so any $\Pi_{1}^{0}(g)$-subclass of $\mathcal{Q}_{g}$, such as $\mathcal{P} \cap \mathcal{Q}_{g}$, must be either empty or perfect.

Definition 3.4. Let $A \subseteq \omega$ be a set and let $f: \omega \rightarrow \omega$ be a function. Let $\mathcal{P}$ be a $\Pi_{1}^{0}$-class. An $A, f$-covering of $\mathcal{P}$ is a map $x \mapsto \sigma_{x}$ from some finite subset $D$ of $A$ such that for all $x \in D,\left|\sigma_{x}\right|>f(x)$, and such that

$$
\mathcal{P} \subset \bigcup_{x \in D}\left[\sigma_{x}\right]
$$

(Compare with [8, Definition 5.1].) 
Lemma 3.5. Suppose that $f$ is a computable function and that $A$ is an infinite set which does not compute a DNR function. Then every countable $\Pi_{1}^{0}$ class has an $A, f$-covering.

Proof. We follow the proof of [8, Theorem 5.2]. We split $A$ into disjoint sets $\left\langle I_{k}\right\rangle$ with each $I_{k}$ of size $2^{k}$ (and, for simplicity, $\max I_{k}<\min I_{k+1}$ ); we let $g(k)=$ $\max _{x \in I_{k}} f(x)$. Inductively, for each $x \in A$ we pick a string $\sigma_{x}$ of length $g(k)$ (if $x \in I_{k}$ ) of minimal plain complexity (which has not been chosen so far). Again, if $\sigma \in 2^{g(k)}$ and $C(\sigma)<k$, then $\sigma=\sigma_{x}$ for some $x \in I_{k}$, so $\mathcal{Q}_{g}$ is disjoint from $\bigcup_{x \in A}\left[\sigma_{x}\right]$.

Now $g \leqslant_{T} A$, so $g$ does not compute a DNR function. So if $\mathcal{P}$ is countable, then $\mathcal{P} \cap \mathcal{Q}_{g}=\emptyset$ and so $\bigcup_{x \in A}\left[\sigma_{x}\right]$ covers $\mathcal{P}$; so by compactness, some finite subcover covers $\mathcal{P}$ and is an $A, f$-covering.

Remark 3.6. We could have used the notion of $h$-complex sets, which is equivalent to being in $\mathcal{Q}_{g}$ (for some $g$ of the same Turing degree as $h$ ) if $h$ has $K$-trivial degree. We could have proceeded with the proof of Proposition 3.1 because the construction of a hypersimple set is consistent with making it $K$-trivial; that is, we could obey the standard cost function. Instead, we use the blunter instruments of lowness (and Arslanov's completeness criterion).

Construction. We enumerate a set $A$ and attempt to meet the requirements $R_{e}$ and $P_{e}$ above. In addition, we make $A$ incomplete by ensuring that it is low; let $N_{e}$ be the $e^{\text {th }}$ lowness requirement, i.e. the requirement which tries to ensure that the halting or not of $\Phi_{e}^{A}(e)$ can be decided given the (unrelativised) halting problem $\emptyset^{\prime}$. The strategy for $N_{e}$ is to build a computable approximation with final value 1 if $\Phi_{e}^{A}(e)$ is defined, and 0 otherwise. Intuitively, $N_{e}$ will be trying to preserve $\Phi_{e}^{A}(e)$ if such a computation is discovered.

We impose finitary restraint between requirements using initialisation. Generally, whenever a requirement receives attention, we initialise all weaker requirements. We also ensure that $A$ is co-infinite by imposing restraint when certain markers move: at stage $s$, let $\left\langle a_{k, s}\right\rangle$ be an increasing enumeration of $\omega \backslash A_{s}$; if $a_{k, s} \neq a_{k, s+1}$, then all requirements $Q_{k}$ (for $Q \in\{R, P, N\}$ ) are initialised. For any requirement $Q_{e}$, we let $r_{s}\left(Q_{e}\right)$ be the last stage before $s$ at which $Q_{e}$ was initialised; this is the restraint imposed on $Q_{e}$ at stage $s$.

We now describe the instructions for all requirements. We describe when a requirement requires attention, and what it does if it receives it. As usual, at every stage, the strongest requirement which requires attention, receives it.

At stage $s$ :

$N_{e}$ : A requirement $N_{e}$ requires attention if it has not received attention since stage $r_{s}\left(N_{e}\right)$, and $A^{\prime}(e) \downarrow[s]$. When it receives attention, it does nothing (except for initialising all weaker requirements).

$P_{e}:$ A requirement $P_{e}$ requires attention if there is no $n \in \operatorname{dom} g_{e, s}$ such that $D_{g_{e}(n)} \subset A_{s}$, and there is some $n \in \operatorname{dom} g_{e, s}$ such that $\min D_{g_{e}(n)}>r_{s}\left(P_{e}\right)$. If it receives attention, then one such $n$ is chosen and the contents of $D_{g_{e}(n)}$ are enumerated into $A$.

$R_{e}$ : A requirement $R_{e}$ first searches for a suitable covering. So if, at stage $s, R_{e}$ has no covering, then it searches for a covering $\left\langle\sigma_{x}\right\rangle_{x \in D}$ which is currently suitable:

- The covering appears by stage $s$ : for all $x \in D, x<s$ and $\left|\sigma_{x}\right|<s$. 
- $D$ is available for diagonalisation: $D \cap A_{s}=\emptyset$.

- $D$ is unrestrained: $\min D>r_{s}\left(R_{e}\right)$.

- This is an $\omega \backslash A_{s}, \varphi_{e}$-covering: $D \subset \operatorname{dom} \varphi_{e, s}$ and for all $x \in D$, $\left|\sigma_{x}\right|>\varphi_{e}(x)$.

- It currently covers $\mathcal{P}_{e}: \mathcal{P}_{e, s} \subseteq \bigcup_{x \in D}\left[\sigma_{x}\right]$.

If such a covering appears, then $R_{e}$ requires attention. If it receives attention, then it picks the covering to work with (and initialises weaker requirements). Note that if later, $R_{e}$ is initialised, then the covering is no longer permitted, it is abandoned, and $R_{e}$ needs to resume the search for a new one.

If a covering $\left\langle\sigma_{x}\right\rangle_{x \in D}$ is already appointed, then $R_{e}$ requires attention if there is some $x \in D$ such that $\Phi_{e}^{\sigma_{x}}(x) \downarrow[s]=0$ and $x \notin A_{s}$. If it receives attention, $R_{e}$ enumerates $x$ into $A$.

\section{Verification.}

Lemma 3.7. The construction is fair: for every requirement $Q_{e}$ there is a stage after which it is never initialised; it requires attention only finitely many times. Also, A is co-infinite.

Proof. The usual proof, by induction on the priority ordering. Suppose that $s^{*}$ is the last stage at which any requirement which is stronger than a requirement $Q_{e}$ ever receives attention (and so no such requirements ask for attention after stage $s^{*}$ ) and by $s^{*}$ all $a_{i, s}, i<e$ have reached a limit. Suppose that there is some stage $s>s^{*}$ such that $a_{e, s} \neq a_{e, s+1}$. By the induction hypothesis, $e$ is the least number with this property; hence $a_{e, s+1}<s$. Then at stage $s$ all of $P_{e}, R_{e}$ and $N_{e}$ are initialised, so we have $r_{t}\left(Q_{e^{\prime}}\right)>a_{e, s+1}$ for all $t>s$ and all requirements $Q_{e^{\prime}}$ which are not stronger than $Q_{e}$. Thus $a_{e, s+1}$ (and any smaller number) is never enumerated into $A$ after stage $s$, so after stage $s, Q_{e}$ is never initialised.

Now depending on the nature of $Q_{e}$, it is easy to check that after it is last initialised, $Q_{e}$ only receives attention finitely often; in the case of $N_{e}$ and $P_{e}$, it is at most once, and in the case of $R_{e}$, it is once for picking a covering $\left\langle\sigma_{x}\right\rangle_{x \in D}$ and then at most once for every $x \in D$.

Lemma 3.8. $A$ is low and hypersimple.

Proof. The requirement $P_{e}$ is met: if $g_{e}$ is total and $\left\langle D_{g_{e}(n)}\right\rangle$ are pairwise disjoint, then there is some $n$ such that $\min D_{g_{e}(n)}$ is greater than the last stage at which $P_{e}$ is initialised; then at some stage, $P_{e}$ acts.

The requirement $N_{e}$ is met: if $s^{*}$ is the last stage at which $N_{e}$ is initialised, and there is some $s>s^{*}$ at which $A_{s}^{\prime}(e)$ converges, then by initialisation, this computation is preserved.

Lemma 3.9. $A$ is not wtt-reducible to any ranked set.

Proof. We show that every requirement $R_{e}$ is met. Suppose that $\mathcal{P}_{e}$ is countable and that $\varphi_{e}$ is total. Since $A$ is low, it is incomplete (and has c.e. degree), so by Arslanov's completeness criterion (see, e.g., 34]), $A$ computes no DNR function. Let $s^{*}$ be the last stage at which $R_{e}$ is initialised, and let $B=\omega \backslash\left(A \cup\left\{n \mid n \leq s^{*}\right\}\right)$, which is of course Turing equivalent to $A$.

So there is some $B, \varphi_{e}$-covering $\left\langle\sigma_{x}\right\rangle_{x \in D}$ of $\mathcal{P}$. Such a covering will be discovered by some stage $s>s^{*}$ (and will be permitted), and so we know that $R_{e}$ picks some 
covering at some stage $s>s^{*}$. Later, $R_{e}$ may diagonalise whenever it needs to, so it is met.

3.2. A ranked set which is not wtt-reducible to a hypersimple set. Here we prove:

Proposition 3.10. There is a c.e. ranked set (indeed of rank 1) which is not wttreducible to any hypersimple set.

We call the set we enumerate $A$. To make $A$ ranked, we make it an initial segment of a scattered, computable linear ordering; it will be the $\omega$-part of an ordering of type $\omega+\left(\omega^{2}\right)^{*}$. This is the "shortest" possible example, because Lemma 1.3 implies that if $\gamma<\omega^{2}$ and $A$ is a c.e. and non-computable initial segment of a computable ordering of type $\omega+\gamma^{*}$, then $A$ is computably isomorphic to a hypersimple set.

The strategy of making $A$ not wtt-reducible to any hypersimple set is similar to that from Section 2.1. Let us remind the reader of the requirements we need to satisfy:

$R_{e}$ : If $\Phi_{e}^{W_{e}}=A$, then $W_{e}$ is not hypersimple.

(Here $\left\langle W_{e}, \Phi_{e}\right\rangle$ is a list of all pairs consisting of a c.e. set and a weak truth-table functional with use function $\varphi_{e}$.) We combine the strategy from Section 2.1 with a priority argument to get the present construction. Again, a requirement $R_{e}$ will choose finite sets of followers $X_{0}^{e}, X_{1}^{e}, X_{2}^{e}, \ldots$ such that $\left|X_{n+1}^{e}\right|>u_{n}^{e}$, where for all $x \in X_{n}^{e}$ we have $\varphi_{e}(x)<u_{n}^{e}$. The sets $X_{n}^{e}$ are placed in the $e^{\text {th }}$ copy of $\omega^{*}$ in the linear ordering we are building, until we decide to attack with some $X_{n}^{e}$; we then move the weaker requirements to build between the elements of $X_{n}^{e}$.

Construction. Each requirement $R_{e}$ defines an increasing sequence $\left\langle u_{n}^{e}\right\rangle$ of natural numbers and finite sets of followers $\left\langle X_{n}^{e}\right\rangle$. When $R_{e}$ is initialised, we abandon its current sequences and start rebuilding from $n=0$. A requirement $R_{e}$ can be in waiting mode or in attack mode. (If $R_{e}$ is initialised, then it is returned to waiting mode.)

At stage $s$, if $R_{e}$ is in waiting mode, then it requires attention if one of the following holds:

(1) no $X_{n}^{e}$ is currently defined;

(2) $X_{0}^{e}, \ldots, X_{n}^{e}$ are currently defined and for all $x \in X_{n}^{e}, \varphi_{e}(x) \downarrow$;

(3) there is some $n$ such that $u_{n}^{e}$ is defined and $\left[u_{n-1}^{e}, u_{n}^{e}\right) \subset W_{e}$.

If $R_{e}$ is in attack mode (with $X_{n}^{e}$ ), then it requires attention if $\Phi_{e}^{W_{e}}$ currently agrees with $A\left\lceil\max X_{n}^{e}+1\right.$.

We let the strongest requirement which requires attention receive it. Suppose that $R_{e}$ receives attention. If $R_{e}$ is in waiting mode, we act according to the cases above.

(1) Let $X_{0}^{e}$ be some finite set of fresh numbers. In the linear ordering $<^{*}$ that we are building, place the elements of $X_{0}^{e}$ immediately to the right of $A_{s}$.

(2) Let $u_{n}=1+\max \left\{\varphi_{e}(x): x \in X_{n}^{e}\right\}$. Let $X_{n+1}^{e}$ consist of $u_{n}+1$ many fresh numbers. In the linear ordering $<^{*}$ that we are building, place the elements of $X_{n+1}^{e}$ immediately to the left of the elements of $X_{n}^{e}$.

(3) Move $R_{e}$ to attack mode with $X_{n}^{e}$.

If $R_{e}$ is attacking with $X_{n}^{e}$, we enumerate the $<^{*}$-leftmost element of $X_{n}^{e} \backslash A_{s}$ into $A$ and initialise all weaker requirements. Maintain $A$ as an initial segment of 
$<^{*}$ by enumerating into $A$ any numbers which lie to the left of the number just enumerated into $A$ (these are the elements of $X_{m}^{e^{\prime}}$ for $e^{\prime}>e$ ).

Verification. It is clear that $<^{*}$ that we built is a computable linear ordering, since we placed any newly chosen followers into $<^{*}$ immediately. It is also clear that the order-type of $A$ in $<^{*}$ is $\omega$, because once an element is enumerated into $A$, no new element is ever placed to its left. Further, it is also clear that the construction is fair, in that every requirement is initialised only finitely many times, because a requirement $R_{e}$ only initialises weaker requirements if it is in attack mode, and then it acts only $\left|X_{n}^{e}\right|$ many times (if it is not later initialised).

Lemma 3.11. The order-type of $<^{*}$ is $\omega+\left(\omega^{2}\right)^{*}$.

Proof. Every requirement $R_{e}$ has three possible eventual outcomes (after it is last initialised):

- $R_{e}$ defines only finitely many sets of followers $X_{n}^{e}$ (because $\varphi_{e}$ is not total) and does not attack. In that case, $R_{e}$ adds only finitely many points to $<^{*}$ and does not change its order-type.

- $R_{e}$ defines infinitely many $X_{n}^{e}$ but never attacks. In that case, it adds to $<^{*}$ an interval of order-type $\omega^{*}$. This happens, for example, whenever $\varphi_{e}$ is total but $W_{e}$ is empty.

- $R_{e}$ eventually attacks with some $X_{n}^{e}$. In this case again $R_{e}$ adds only finitely many points to $<^{*}$.

Whenever a requirement $R_{e}$ attacks and initialises weaker requirements, finitely many points are added to $A$, but the picture for stronger portions of $<^{*}$ doesn't change.

Lemma 3.12. A is not wtt-reducible to any hypersimple set.

Proof. Suppose for contradiction that $W_{e}$ is hypersimple and that $\Phi_{e}^{W_{e}}=A$. Let $s_{0}$ be the last stage at which $R_{e}$ is initialised. Since $\varphi_{e}$ is total, $R_{e}$ can always define new sets of followers $X_{n}^{e}$, and since $W_{e}$ is hypersimple, we eventually discover some $n$ such that $\left[u_{n-1}, u_{n}\right) \subset W_{e}$; so eventually, at some stage $s_{1}>s_{0}, R_{e}$ attacks with some $X_{n}^{e}$. We are then later allowed to change $A\left\lceil\max X_{n}^{e}+1\right.$ more times than the possible future configurations for $W_{e} \uparrow u_{n}$, which leads to the desired contradiction.

\section{Computable Lipschitz Reductions AND ARRAy COMPutable DEgREes}

4.1. Multiple permitting I: Common upper bounds. Here we prove Proposition 1.8. If $\mathbf{d}$ is c.e. and array non-computable, then there are left-c.e. reals $\alpha_{0}$ and $\alpha_{1}$ in $\mathbf{d}$ which do not have a common upper bound in the ibT-degrees of left-c.e. reals. This is obtained by adding coding and multiple permitting to the construction of two left-c.e. reals with no common upper bound in the cl-degrees from [35, 5, which we now rephrase for our purposes.

The main idea of the Yu-Ding construction is that if $\beta$ is a left-c.e. real which ibT-computes both $\alpha_{0}$ and $\alpha_{1}$, then alternatingly adding little bits to $\alpha_{0}$ and $\alpha_{1}$ (drip-feeding them) is sufficient to drive $\beta$ to be too large. Furthermore, we can computably reserve, for each requirement, an interval of its own, which is sufficient for its purposes. Here are the details. 
Suppose that $\Gamma_{0}$ and $\Gamma_{1}$ are ibT reductions and that $\beta$ is a left-c.e. real. For all such triples, we need to meet the requirement

$$
R_{\Gamma_{0}, \Gamma_{1}, \beta} \text { : Either } \Gamma_{0}^{\beta} \neq \alpha_{0} \text { or } \Gamma_{1}^{\beta} \neq \alpha_{1} \text {. }
$$

We view left-c.e. reals as both infinite binary sequences and as the corresponding elements in the Euclidean interval $[0,1]$ (via binary expansion). Addition means addition in the real field $\mathbb{R}$.

To meet the requirement $R=R_{\Gamma_{0}, \Gamma_{1}, \beta}$, we describe a family of modules, each indexed by an interval of natural numbers. Let $a \leqslant b<\omega$. A stage $s$ is expansionary for the $[a, b)$-module (for $R$ ) if for both $i=0,1$ we have $\Gamma_{i}^{\beta_{s}} \supset \alpha_{i, s} \uparrow b$. The instructions for the module are:

Repeat the following $2^{b-a}-1$ times:

(1) At the next expansionary stage, add $2^{-b}$ to $\alpha_{0}$.

(2) At the next expansionary stage, add $2^{-b}$ to $\alpha_{1}$.

At the end, wait for the next expansionary stage and then return.

To meet the requirement $R$, we run the $\left[k, k+2^{k}\right)$-module for $R$ for some $k$. We will shortly argue that this module cannot return, and so it must get stuck waiting for some expansionary stage, and so $R$ is met. It is easy to see that assuming that we start with $\alpha_{i} \uparrow[a, b)=0^{b-a}$, the $[a, b)$-module for any requirement only makes changes in $\alpha_{i} \uparrow[a, b)$ (for both $i=0,1$ ) and so if for distinct requirements we run modules on disjoint intervals, there is no interaction between the requirements and so we can meet them all.

The verification relies on the following lemma. For this lemma, we also think of a finite binary string as a natural number (via binary expansion 6 ).

Lemma 4.1. Suppose that $\alpha_{0, t_{0}} \uparrow[a, b)=\alpha_{1, t_{0}} \uparrow[a, b)=0^{b-a}$. Suppose that at stage $t_{0}$, an $[a, b)$-module for $R$ begins and returns at a stage $t_{1}$. Then

$$
\beta_{t_{1}} \uparrow a-\beta_{t_{0}} \uparrow a \geqslant b-a .
$$

Proof. By induction on $b-a$. If $b=a$ there is nothing to prove. Assume the lemma holds for $n$, and let $a<b<\omega$ such that $b-a=n+1$. The key observation is that the $[a, b)$-module consists of three parts:

- running the $[a+1, b)$-module;

- running one iteration of adding $2^{-b}$ to $\alpha_{0}$ and then $\alpha_{1}$ (and waiting for expansionary stages);

- running the $[a+1, b)$-module again.

We also note that both times that we run the $[a+1, b)$-module, we start with $\alpha_{i} \uparrow[a+1, b)=0^{n}$ : at the first time, by assumption of the lemma, and by the second, because when the first $[a+1, b)$-module halts we have $\alpha_{i} \uparrow[a, b)=01^{n}$ for both $i$; we then add $2^{-b}$ which makes $\alpha_{i} \uparrow[a, b)=10^{n}$.

Let $s_{0}$ be the stage at which the first $[a+1, b)$-module returns, and let $s_{1}$ be the stage at which the second one begins. By induction, therefore, we have

$$
\beta_{s_{0}} \uparrow a+1-\beta_{t_{0}} \uparrow a+1 \geqslant n
$$

and

$$
\beta_{t_{1}}\left\lceil a+1-\beta_{s_{1}} \uparrow a+1 \geqslant n\right.
$$

\footnotetext{
${ }^{6}$ With the understanding that a prefix of 0s may occur in the binary digits of a number; so the correspondence is not $1-1$.
} 
Between $s_{0}$ and $s_{1}$ we have a change in $\alpha_{0}(a)$, which forces a change in $\beta\lceil a+1$ by the next expansionary stage, and then a change in $\alpha_{1}(n)$, which forces another change in $\beta\lceil a+1$. Each of these changes adds at least 1 to $\beta\lceil a+1$. Thus in total,

$$
\beta_{t_{1}}\left\lceil a+1-\beta_{t_{0}}\lceil a+1 \geqslant 2(n+1)\right.
$$

which implies that

$$
\beta_{t_{1}}\left\lceil a-\beta_{t_{0}}\lceil a \geqslant n+1\right.
$$

as required.

This concludes the verification: the $\left[k, k+2^{k}\right)$-module can never return because that would force $\beta\left\lceil k \geqslant 2^{k}\right.$, which is impossible.

Now to add multiple permitting, we use the original definition of array computability from [19]: A c.e. degree $\mathbf{d}$ is array non-computable iff for all (or some) computable partitions $\left\langle F_{n}\right\rangle$ of $\omega$ of increasing size, there is a c.e. set $D \in \mathbf{d}$ such that for all c.e. sets $W$ there are infinitely many $n$ such that $W \uparrow F_{n}=D \uparrow F_{n}$. Later, we will calculate the desired size of $F_{n}$. Assuming this has been done, fix some $D \in \mathbf{d}$ which satisfies the definition for this $\left\langle F_{n}\right\rangle$. To get sufficiently many permissions, a requirement $R=R_{\Gamma_{0}, \Gamma_{1}, \beta}$ as above enumerates an auxiliary c.e. set $W_{R}$ and ties its actions to permissions from $F_{n}$.

Again, a module for requirement $R$ will operate on some interval $[a, b)$; the notion of an expansionary stage for a module for $R$ on an interval $[a, b)$ is defined as before. For such a module, we will assign some $n$ so that $\left|F_{n}\right|>2\left(2^{b-a}-1\right)$. Note that we choose distinct $n$ 's for each module for $R$. To request permission, the module picks some $x \in F_{n}$ which is not yet in $W_{R}$ and enumerates it into $W_{R}$. Permission is received when at a later stage, $x$ enters $D$. The standard modus operandi for multiple permitting is used: if some $x \in F_{n}$ enters $D$ before it is enumerated into $W_{R}$, then $F_{n}$ can be made incorrect for permitting by withholding $x$ from ever entering $W_{R}$, so we assume this never happens.

The new module follows the following instructions:

Repeat the following $2^{b-a}-1$ times:

(1) Wait for an expansionary stage; then request permission. When permission is received, add $2^{-b}$ to $\alpha_{0}$.

(2) Wait for another expansionary stage; then request permission. When permission is received, add $2^{-b}$ to $\alpha_{1}$.

At the end, wait for the next expansionary stage and then return.

Note that the choice of $n$ ensures that we can always request a new permission, as the module above requests at most $2\left(2^{b-a}-1\right)$ permissions and so we never get $F_{n} \subseteq W_{R}$.

The overall construction is as expected. For every $R$ we now pick an infinite set of intervals of the form $\left[k, k+2^{k}\right.$ ) and run modules on each interval separately (all of these modules together enumerate $W_{R}$ though). We ensure that these intervals are pairwise disjoint and further that the intervals used by different requirements are also pairwise disjoint. Also, to code in $D$, we fix a computable set $C$ which is disjoint from every interval used by any requirement. Let $c_{n}$ be the $n^{\text {th }}$ element of $C$; we declare that for both $i=0,1, \alpha_{i}\left(c_{n}\right)=1$ iff $n \in D$. Since $D$ is a c.e. set and 
all modules only change $\alpha_{i}$ on their intervals, we still see that both $\alpha_{i}$ are left-c.e. reals.

To conclude the construction, we need to specify the required size of $F_{n}$ so that we can assign, for every requirement $R$, almost every $n$ as a permitting number for some module working for $R$. So after we specify the coding location set $C$ and assign the intervals for every requirement, we define $\left|F_{n}\right|$ to be large enough so that for every $m \leqslant n$, for each of the first $m$ requirements, if $[a, b)$ is the $n^{\text {th }}$ interval assigned to $R$, then $\left|F_{n}\right|>2\left(2^{b-a}-1\right)$, so $F_{n}$ can be assigned as a permitting set for the $[a, b)$-module for $R$.

For the verification, we note that Lemma 4.1 holds for the new construction, with the same proof. Thus, for any requirement $R$, no $\left[k, k+2^{k}\right)$-module for $R$ ever returns. The standard permitting argument now shows that it cannot be that every module for some requirement $R$ gets stuck waiting for permission; since for almost all $n, F_{n}$ is assigned as a permitting set for some module for $R$, there is some such $n$ such that $W_{R} \uparrow F_{n}=D \uparrow F_{n}$ and so the module for which $F_{n}$ is assigned is never stuck waiting for permission. As a result, it must get stuck waiting for an expansionary stage, and so the requirement $R$ is met.

It is clear by coding that $D \leqslant_{T} \alpha_{0}, \alpha_{1}$. The next lemma concludes the verification.

Lemma 4.2. $\alpha_{0}, \alpha_{1} \leqslant_{T} D$.

Proof. Fix $i<2$. Let $x<\omega$. To decide $\alpha_{i}(x)$ with oracle $D$, it depends in which part of $\omega x$ lies. If $x \in C$, then we can easily calculate the $n$ such that $x=c_{n}$ and then consult $D$ for the value of $\alpha_{i}(x)$. Otherwise, $x$ belongs to some interval $[a, b)$ on which a module for some requirement $R$ is working. This requirement is assigned some permitting set $F_{n}$. Wait for a stage $s$ at which $D_{s} \uparrow F_{n}=D \uparrow F_{n}$. Then after stage $s, \alpha_{i} \uparrow[a, b)$ is fixed and so $\alpha_{i, s}(x)=\alpha_{i}(x)$.

4.2. Failure of multiple permitting I: Common upper bounds. Here we prove Proposition 1.9, if $\alpha_{0}$ and $\alpha_{1}$ are left-c.e. reals which have array computable (Turing) degrees, then $\alpha_{0}$ and $\alpha_{1}$ have a common upper bound in the cl-degrees. The idea is that we can approximate $\alpha_{0}$ and $\alpha_{1}$ with the mind-changes bound growing as slowly as we like. This follows from the characterisation of array computability as uniform total $\omega$-c.e.-ness (from [19]): A c.e. degree $\mathbf{d}$ is array computable iff for every computable order $h$, every $f \leqslant_{T} \mathbf{d}$ has an $h$-c.e. approximation. We can then use this slow bound to build a left-c.e. real $\beta$ which changes somewhere on its first $n$ digits whenever either $\alpha_{0}$ or $\alpha_{1}$ do so.

Again we think of these reals as elements of the interval $[0,1]$. A request that $\beta \uparrow n$ change is ensured by adding a quantity of $2^{-n}$ to $\beta$. If the number of requests for a $\beta\lceil n$ change does not exceed a number $g(n)$, and if

$$
\sum_{n \geqslant 1} g(n) 2^{-n}<1
$$

then we can construct a left-c.e. real that changes appropriately whenever we ask it to. In our case, a request to change $\beta \uparrow n$ is made whenever we believe a new value for $\alpha_{0} \uparrow n$ or $\alpha_{1} \uparrow n$, so if the number of initial segments of length $n$ which we believe for $\alpha_{i}$ is at most $g(n) / 2$, then the plan will work. So all we need to do is to 
fix some computable order $g$ which grows sufficiently slowly - say one bounded by a polynomial - so that (4.1) holds, and approximate the functions $n \mapsto \alpha_{i}\lceil n$ in a $g(n) / 2$-c.e. way, which is possible by assumption.

There is not much to add to give a formal construction. Fix a computable order $g$ that satisfies (4.1). For $i=0,1$, fix computable functions $f_{i, s}(n)=f_{i}(s, n)$ such that for all $n, f_{i, s}(n)$ is a binary string of length $n$,

$$
\#\left\{s: f_{i, s+1}(n) \neq f_{i, s}(n)\right\} \leqslant g(n) / 2
$$

(if $g(n)<2$, then $f_{i, s}(n)$ has to be constant in $s$ ), and

$$
\lim _{s} f_{i, s}(n)=\alpha_{i} \uparrow n
$$

We define a left-c.e. real $\beta$ : start with $\beta_{0}=0$, and let

$$
\beta_{s+1}=\beta_{s}+\sum\left\{2^{-n}: f_{i, s+1}(n) \neq f_{i, s}(n)\right\} .
$$

The fact that $g$ satisfies (4.1) and the restriction on the number of mind-changes for the $f_{i}$ imply that $\beta=\lim _{s} \beta_{s}<1$, so $\beta$ is well defined.

Lemma 4.3. $\alpha_{i} \leqslant \mathrm{ibT} \beta$.

Proof. If $s$ is a stage such that $\beta_{s} \uparrow n=\beta\lceil n$, then we never, after stage $s$, add a quantity of $2^{-n}$ to $\beta$. This implies that for all $t>s, f_{i, t}(n)=f_{i, s}(n)$. Thus $f_{i, t}(n)=\alpha_{i}\lceil n$.

Remark 4.4. We didn't use the fact that the $\alpha_{i}$ are left-c.e. reals; the proof works for any sets which have array computable c.e. degrees because approximations such as the $f_{i}$ are available for all sets in those degrees.

4.3. Multiple permitting II: Random reals. In this section we prove Proposition 1.10 if $\mathbf{d}$ is c.e. and array non-computable, then there is some left-c.e. real $\alpha \in \mathbf{d}$ which is not ibT-reducible to any random left-c.e. real.

As before, we only add multiple permitting and coding to a construction of a left-c.e. real which is not ibT-reducible to any random left-c.e. real. We give a simpler construction than the one presented in [7, which we now describe.

We build a left-c.e. real $\alpha$ and try to meet the requirements

$$
R_{\Gamma, \beta} \text { : If } \Gamma^{\beta}=\alpha \text {, then } \beta \text { is not random. }
$$

Here $\Gamma$ ranges over all ibT functionals and $\beta$ ranges over all left-c.e. reals. To meet $R$, we will run infinitely many modules, where the $n^{\text {th }}$ module enumerates a finite set of strings $U_{n}$ of measure at most $2^{-n}$, such that if $\Gamma^{\beta}=\alpha$, then $\beta \in U_{n}$. Thus together, all modules for $R$ enumerate a Martin-Löf test which covers $\beta$, and so $\beta$ is not random.

In the previous construction, we used the leeway we had in the play between two left-c.e. reals to drive a potential common upper bound to be too large. Here we only have one real $(\alpha)$ to play with, and the role of the second real is taken by the element of the Martin-Löf test being enumerated. This is much more limited because the restriction on the size of the enumerated set of strings is much stricter than that on the enumeration of the left-c.e. real. 


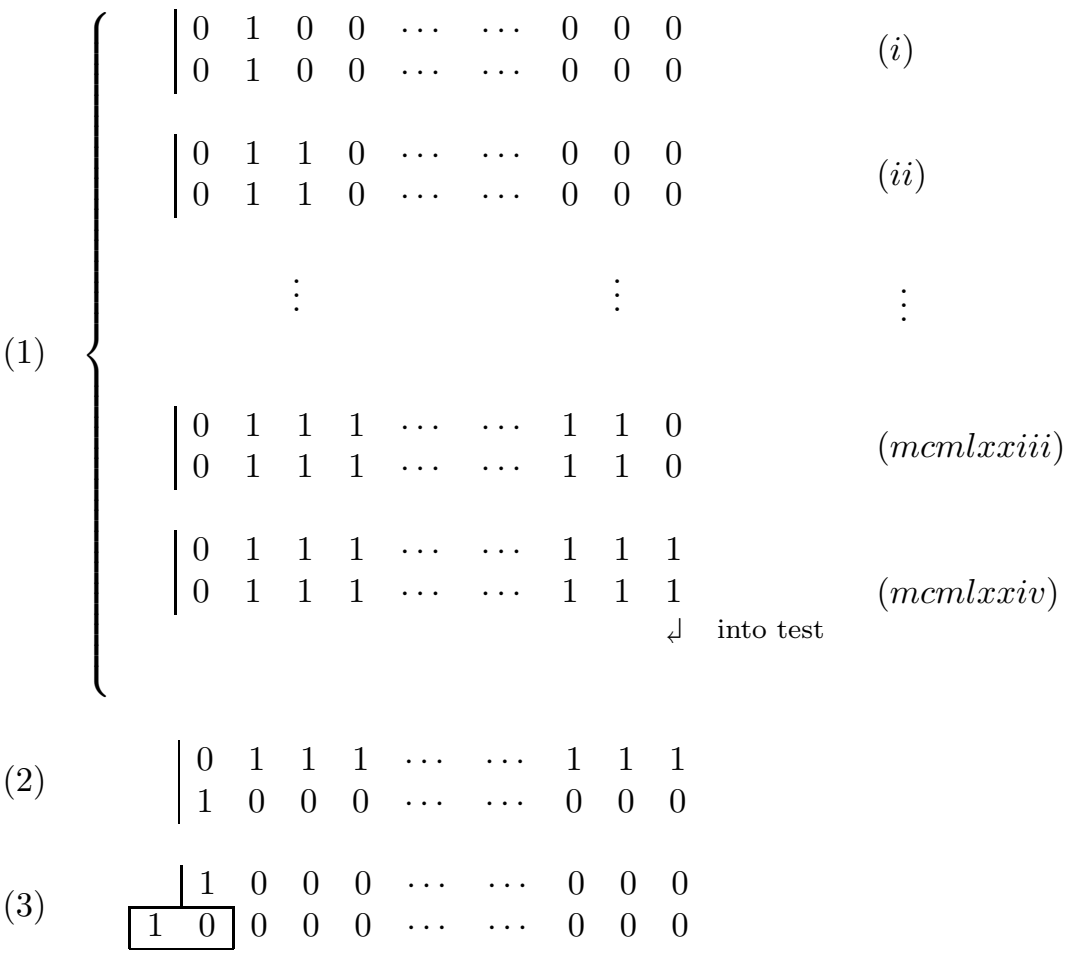

Figure 1. The 2-module

The modules are built by recursion from smaller and smaller building blocks. Consider, for example, the following module, which only changes $\alpha$ from the $a^{\text {th }}$ digit, and makes $\beta \uparrow a+1 \geqslant 2 \sqrt{7}$ As in the Yu-Ding and Barmpalias-Lewis papers, assume (for now) that $\beta$ is playing its optimal strategy, which is adding the minimal amount which is necessary to match $\alpha$ 's movements.

$$
\begin{aligned}
& \text { Set } \alpha(a+1)=1 ; \text { wait for } \beta(a+1)=1 . \\
& \text { Set } \alpha(a+2)=1 \text {; wait for } \beta(a+2)=1 .
\end{aligned}
$$

$$
(\text { mcmlxxiv }) \quad \text { Set } \alpha(a+1974)=1 \text {; wait for } \beta(a+1974)=1 \text {. }
$$

(2) Enumerate $0^{a+1} 1^{1974}$ into the test element; wait for $\beta=0^{a} 10^{\omega}$.

(3) Add $2^{-a-1975}$ to $\alpha$, thus setting $\alpha=0^{a} 10^{\omega}$. Wait for $\beta=0^{a-1} 10^{\omega}$.

The cost, in terms of the measure of strings enumerated into the test element, is $2^{-a-1975}$; as 1975 approaches infinity, we can make the cost as low as we like. Figure 1 shows the state of $\alpha$ and $\beta$ at the end of every stage of the 2 -module. The first digit to the right of the horizontal line is the $a^{\text {th }}$. At the end of the first step, we indicate the initial segment of $\beta$ which is enumerated into the test element at the second step of the module; and at the end we indicate the amount (2, which is 10 in binary) added to $\beta \uparrow a+1$.

\footnotetext{
${ }^{7}$ As before, we also think of finite binary strings as natural numbers via their binary expansion, with the understanding that a prefix of 0s may occur in the binary digits of a number.
} 


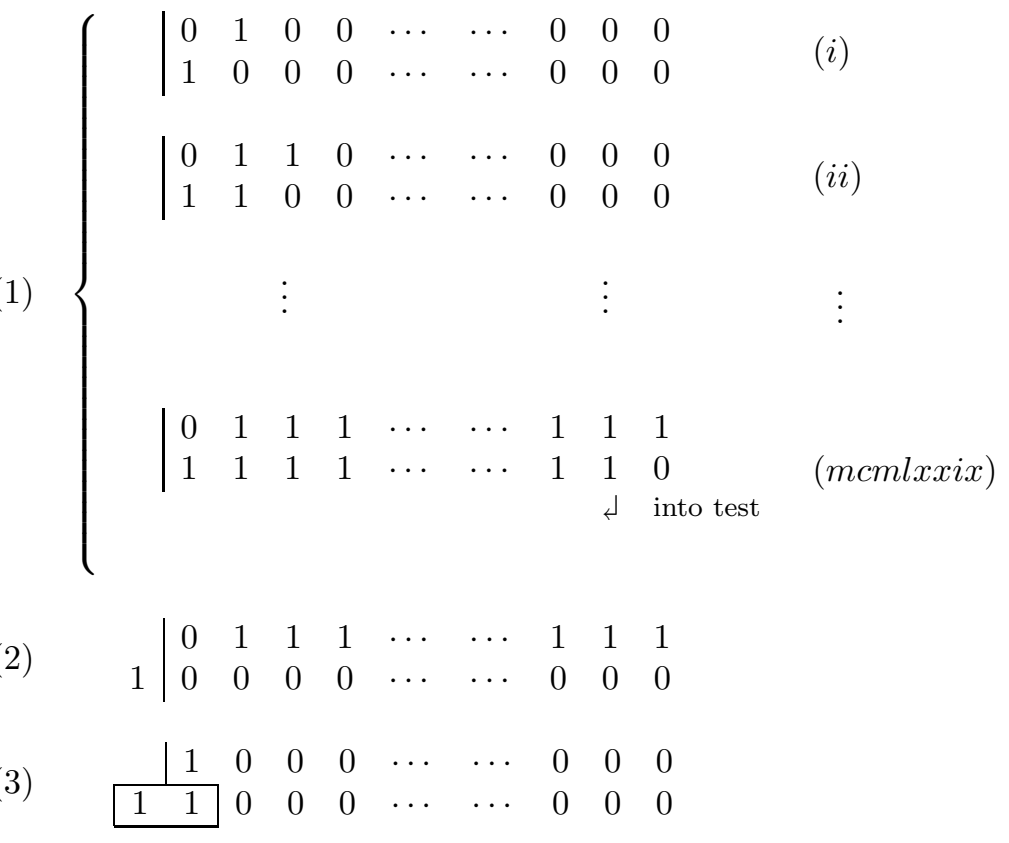

Figure 2. The 3-module

Now this module can be iterated to make $\beta\lceil a+1 \geqslant 3$ :

Run the 2-module from point $a+1$, to get $\alpha(a+1)=1$ and $\beta(a)=1$.

Run the 2-module from point $a+2$, to get $\alpha(a+2)=1$ and $\beta(a+1)=1$.

Run the 2 -module from point $a+1979$
to get $\alpha(a+1979)=1$ and $\beta(a+1978)=1$.

(2) Enumerate $0^{a} 1^{1979}$ into the test element; wait for $\beta=0^{a-1} 10^{\omega}$.

(3) Set $\alpha=0^{a} 10^{\omega}$. Wait for $\beta=0^{a-1} 110^{\omega}$.

Again the cost can be kept down by making the number 1980 large, and then keeping the cost of every recursive call of the 2-module low as well. Figure 2 follows the 3 -module.

For the 4-module (Figure 3), note the growing distance between the last point of change in $\alpha$ and the end of the string of 1's in $\beta$ which goes into the test. This distance, according to our calculations below, is bounded by $n$, the level of the module.

We turn to formally describe the modules and investigate their properties, without assuming anything about the behaviour of the opponent.

The module $M_{R}(a, n, \varepsilon)$ is indexed by: $a$, the bit of $\alpha$ where it begins acting; $n$, the level of the module; and $\varepsilon$, the bound on the measure of the c.e. open set $U_{M}$ which the module enumerates. 


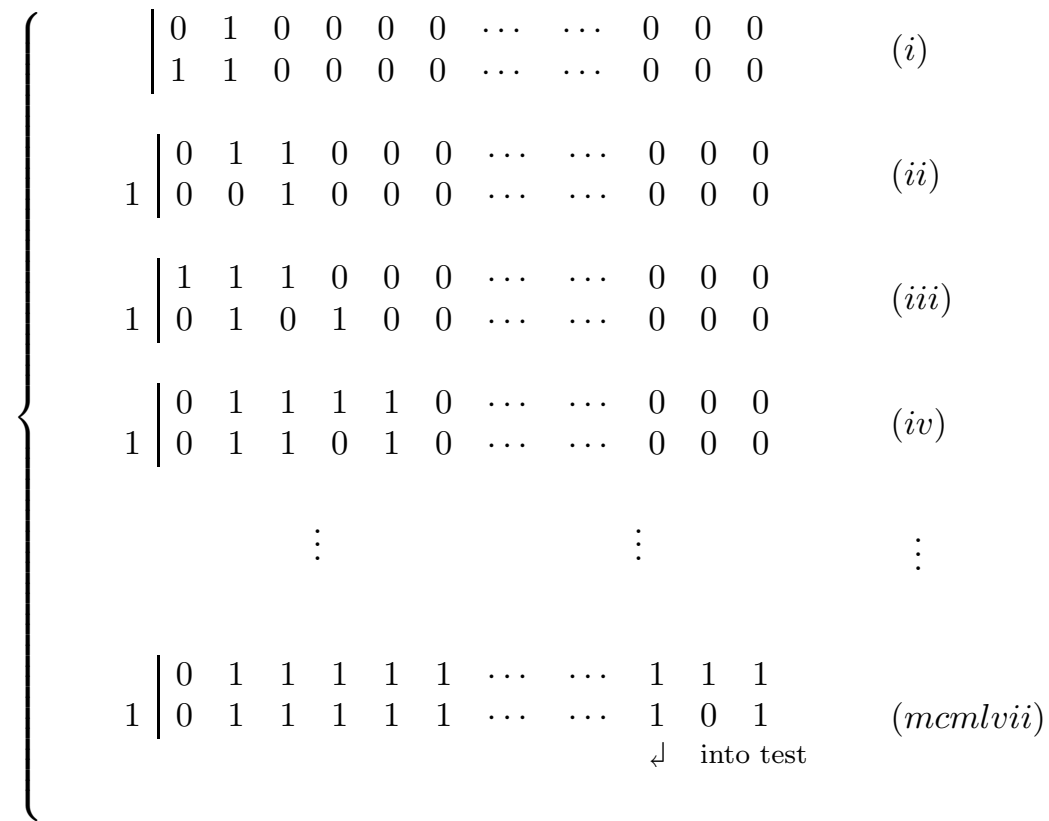

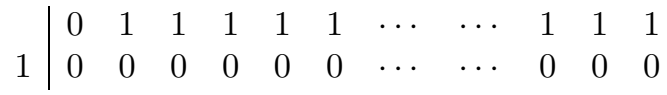

$$
\begin{aligned}
& \begin{array}{|ll|lllllllllll}
\multicolumn{1}{l}{} & & 1 & 0 & 0 & 0 & 0 & 0 & \cdots & \cdots & 0 & 0 & 0 \\
\hline 1 & 0 & 0 & 0 & 0 & 0 & 0 & 0 & \cdots & \cdots & 0 & 0 & 0
\end{array}
\end{aligned}
$$

FiguRe 3. The 4-module

By induction on stages, define the notion of an expansionary stage for the requirement $R=R_{\Gamma, \beta}: 0$ is expansionary, and if $s$ is an expansionary stage for $R$, and $x$ is the largest number mentioned at stage $s$ (or before), then the next expansionary stage is the least stage $t$ at which $\Gamma^{\beta_{t}} \supseteq \alpha_{t}\lceil x$.

The module $M_{R}(a, 1, \varepsilon)$ is:

Wait for an expansionary stage, then add $2^{-a-1}$ to $\alpha$; wait for another expansionary stage, and return with $U_{M}=\emptyset$.

For $n>1$, the module $M_{R}(a, n, \varepsilon)$ is:

Let $b$ be the least $b>a$ such that $2^{-b}<\varepsilon / 2$; let $\varepsilon^{\prime}=\varepsilon /(2(b+n-a))$.

(1) For $k=a+1, a+2, \ldots, b+n$, call $M_{R}\left(k, n-1, \varepsilon^{\prime}\right)$, and add the returned set into $U_{M}$.

(2) Add the current version of $\beta\left\lceil b\right.$ into $U_{M}$, and wait for a stage at which $\beta\lceil b$ changes.

(3) Wait for an expansionary stage, then add $2^{-b-n-1}$ to $\alpha$. Wait for another expansionary stage, and return $U_{M}$. 
We verify some properties of these modules, which will lead to the full construction.

Lemma 4.5. A module $M_{R}(a, n, \varepsilon)$ does not change $\alpha \uparrow a$. Indeed, there is a computable function $c(a, n, \varepsilon)$ such that for all $R, a, n$ and $\varepsilon$, if $c=c(a, n, \varepsilon)$, and a module $M=M_{R}(a, n, \varepsilon)$ starts at stage $s$ with $\alpha_{s}\left\lceil[a, c)=0^{c-a}\right.$, then throughout its run, $M$ changes only $\alpha \uparrow[a, c)$, and if it returns at a stage $t$, then $\alpha_{t} \uparrow[a, c)=10^{c-a-1}$.

Proof. By induction on $n$. The module $M_{R}(a, 1, \varepsilon)$ only changes $\alpha(a)$ from 0 to 1 , so we can let $c=a+1$. For $n>2$, we can calculate $b$ (and $\varepsilon^{\prime}$ ) as in the instructions for the module, and let

$$
c(a, n, \varepsilon)=\max \left\{b+n, c\left(k, n-1, \varepsilon^{\prime}\right): k \in[a+1, \ldots, b+n]\right\} .
$$

Let $c=c(a, n, \varepsilon)$. Since $c \geqslant c\left(k, n-1, \varepsilon^{\prime}\right)$ for all $k \in[a+1, \ldots, b+n]$, if we start with $\alpha_{s} \uparrow[a, c)=0^{c-a}$, then by induction, after $m$ iterations of part (1) of the module $M_{R}(a, n, \varepsilon)$, we have $\alpha \uparrow[a, c)=01^{m} 0^{c-a-m-1}$ and so at the end of part (1) we get $\alpha \uparrow[a, c)=01^{b+n-a} 0^{c-b-n-1}$. At part (2) of the module, $\alpha\lceil[a, c)$ doesn't change, and at part (3), we set $\alpha\left\lceil[a, c)=10^{c-a-1}\right.$ as is promised.

Lemma 4.6. The measure of the set of strings $U_{M}$ enumerated by a module $M_{R}(a, n, \varepsilon)$ is at most $\varepsilon$.

Proof. By a pretty easy induction on $n$.

Again for the next lemma, we also think of finite binary strings as natural numbers.

Lemma 4.7. Suppose that a module $M_{R}(a, n, \varepsilon)$ starts running at stage $s$ (with $\alpha_{s} \uparrow[a, c)=0^{c-a}$, c as above) and returns at stage $t$. Then

$$
\beta_{t}\left\lceil a+1-\beta_{s}\lceil a+1 \geqslant n .\right.
$$

Proof. This goes by induction on $n$. The base case $n=1$ is easy: if $M_{R}(a, 1, \varepsilon)$ starts running at stage $s$ and $\alpha_{s}(a)=0$, then the module changes $\alpha_{s}(a)$ to 1 , and so by the next expansionary stage we get a change in $\beta\lceil a+1$, which implies that $\beta_{t}\left\lceil a+1-\beta_{s}\lceil a+1 \geqslant 1\right.$.

Now assume that the lemma is proved for some $n \geqslant 1$. Assume that we start running $M_{R}(a, n+1, \varepsilon)$ at stage $s_{a}$ with $\alpha_{s_{0}}\left\lceil[a, c)=0^{c-a}\right.$ (where $c=c(a, n+1, \varepsilon)$ ). Calculate the associated $b$ and $\varepsilon^{\prime}$. For $k=a+1, \ldots, b+n+1$, let $s_{k}$ be the stage at which the recursive call of $M_{R}\left(k, n, \varepsilon^{\prime}\right)$ returns. By induction, we have, for all such $k$,

$$
\beta_{s_{k}}\left\lceil k+1-\beta_{s_{k-1}}\lceil k+1 \geqslant n .\right.
$$

For any $m<\omega$, let $Q_{m}=\left\{z 2^{-m}: z \in \mathbb{Z}\right\}$ (see Figure 4). For $\gamma \in(-\infty, \infty)$, let $\llcorner\gamma\lrcorner_{m}$ be the greatest element of $Q_{m}$ which is not greater than $\gamma$. Then for $\gamma \in 2^{\omega}$, again identified with an element of $[0,1]$, we have $\gamma\left\lceil m=2^{m}\llcorner\gamma\lrcorner m\right.$. Also, for $\gamma<\delta$, let

$$
d_{m}(\gamma, \delta)=2^{m}\left(\llcorner\delta\lrcorner_{m}-\llcorner\gamma\lrcorner_{m}\right)=\#\left(Q_{m} \cap(\gamma, \delta]\right) .
$$

The induction hypothesis thus says that

$$
d_{k+1}\left(\beta_{s_{k-1}}, \beta_{s_{k}}\right) \geqslant n \text {. }
$$

Let $\beta^{*}=\left\llcorner\beta_{s_{a}}\right\lrcorner_{a+1}+n 2^{-a-1}$. So $\beta^{*} \in Q_{a+1}$. 


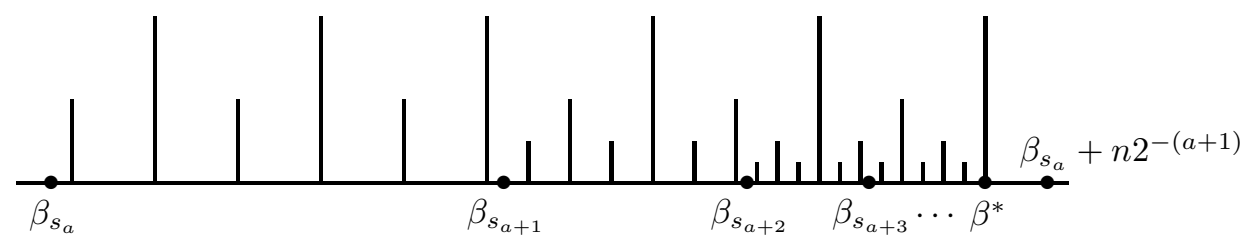

FiguRE 4. The longest lines represent elements of $Q_{a+1}$, the next longest lines elements of $Q_{a+2}$, etc. In this example, $n=6$.

By induction on $k=a, \ldots, b+n+1$, we show that

$$
d_{k+1}\left(\beta_{s_{k}}, \beta^{*}\right) \leqslant n .
$$

For $k=a$ this is immediate. If $k>a$ and (4.3) is true for $k-1$, then because $\beta^{*}$ is in $Q_{k}$, for every $x \in\left(Q_{k+1} \backslash Q_{k}\right) \cap\left(\beta_{s_{k-1}}, \beta^{*}\right]$ there is some $y>x$ in $Q_{k} \cap\left(\beta_{s_{k-1}}, \beta^{*}\right]$ and so

$$
d_{k+1}\left(\beta_{s_{k-1}}, \beta^{*}\right) \leqslant 2 n .
$$

Together with (4.2) we get (4.3) for $k$; see Figure 5

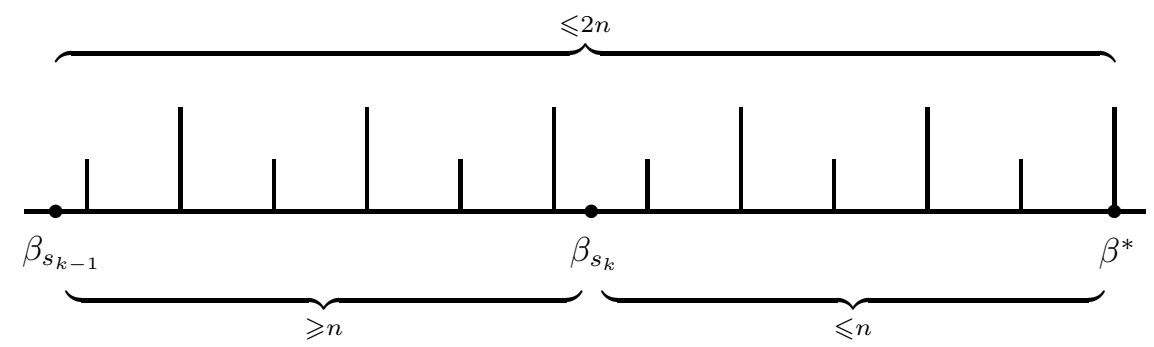

Figure 5. The longer lines represent elements of $Q_{k}$ while the shorter lines represent elements of $Q_{k+1} \backslash Q_{k}$. Again in this example, $n=6$.

At the end, we get $d_{a+b+2}\left(\beta_{s_{b+n+1}}, \beta^{*}\right) \leqslant n$, so $\beta^{*}-\beta_{s_{b+n+1}} \leqslant(n+1) 2^{-b-n-2}$. By Cantor's theorem, $2^{n} \geqslant n+1$, so $\beta^{*}-\beta_{s_{b+n+1}} \leqslant 2^{-b-2}$ (see Figure 6 ). It follows that $\beta^{*}\left\lceil b=\beta_{s_{b+n+1}}\lceil b\right.$.

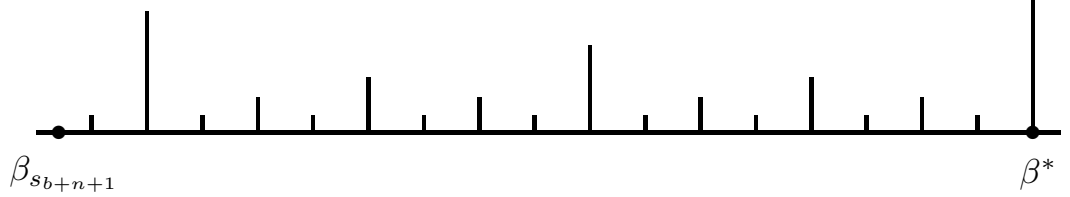

Figure 6 . Even though $d_{b+n+2}\left(\beta_{s_{b+n+1}}, \beta^{*}\right)$ may be $n$ (in this example, $n \geqslant 17$ ), we see that $\beta^{*}$ is the only element of $Q_{b+2}$ between $\beta_{s_{b+n+1}}$ and $\beta^{*}$ itself. Again the shortest lines represent elements of $Q_{b+n+2}$, the next shortest ones elements of $Q_{b+n+1}$, etc. 
So at the end of step (2) of the module $M_{R}(a, n+1, \varepsilon)$, say at a stage $t_{0}$, we have $\beta_{t_{0}}>\beta^{*}$, so $d_{a+1}\left(\beta_{s_{a}}, \beta_{t_{0}}\right) \geqslant n$; in other words, $\beta_{t_{0}}\left\lceil a+1-\beta_{s_{a}}\lceil a+1 \geqslant n\right.$. At step (3) of the module, we change $\alpha(a)$ and by the next expansionary stage, we have a change in $\beta\lceil a+1$, which adds at least 1 to $\beta\lceil a+1$. So if the module returns at stage $t_{1}$, we have

$$
\beta_{t_{1}}\left\lceil a+1-\beta_{s_{a}}\lceil a+1 \geqslant n+1\right.
$$

as required.

The construction is now clear: we partition $\omega$ into disjoint intervals, all of the form $\left[a, c\left(a, 2^{a+1}, 2^{-n}\right.\right.$ !). A requirement $R$ will be assigned infinitely many such intervals and run $M_{R}\left(a, 2^{a+1}, 2^{-n}\right)$ on the $n^{\text {th }}$ interval assigned to $R$. By Lemma 4.7. none of these modules can return, because we cannot have $\beta\left\lceil a+1 \geqslant 2^{a+1}\right.$. If the hypothesis $\Gamma^{\beta}=\alpha$ of $R$ holds, then the module cannot ever be stuck waiting for an expansionary stage, and so it must get stuck waiting for $\beta$ to avoid the set of intervals $U_{M}$ which it enumerates. In other words, if the $n^{\text {th }}$ module for $R$ enumerates $U_{n}$, and $\Gamma^{\beta}=\alpha$, then $\beta \in U_{n}$ as required; so $\beta$ is not random.

Finally, we can add multiple permitting to this construction to get the proof of Proposition 1.10. This is done exactly as in section 4.1, so we describe the proof swiftly. Let $\mathbf{d}$ be a c.e., array non-computable degree. Partition $\omega$ into an infinite computable coding set $C$ and pairwise disjoint intervals of the form $\left[a, c\left(a, 2^{a+1}, 2^{-n}\right)\right.$ ) (all disjoint from $C$ ). Assign to every requirement $R$ infinitely many intervals, where the $n^{\text {th }}$ interval assigned to $n$ is of the form $\left[a, c\left(a, 2^{a+1}, 2^{-n}\right)\right)$. For every module $M_{R}(a, n, \varepsilon)$, calculate the total number $p(a, n, \varepsilon)$ of stages at which the module changes $\alpha$. Now partition $\omega$ into a very strong array ${ }^{8}\left\langle F_{n}\right\rangle$, where for every requirement, for almost every $n,\left|F_{n}\right|>p\left(a, 2^{a+1}, 2^{-n}\right)$ (where the $n^{\text {th }}$ interval for $R$ is $\left.[a, c)\right)$. Let $D \in \mathbf{d}$ be a c.e. $\left\langle F_{n}\right\rangle$-a.n.c 9

Every requirement $R$ enumerates an auxiliary c.e. set $W_{R}$. To request permission, a module $M=M_{R}\left(a, 2^{a+1}, 2^{-n}\right)$ enumerates some new $x \in F_{n}$ into $W_{R}$. Permission is later received when $x$ appears in $D$. The new instructions for the modules are as for the original ones, except that every change in $\alpha$ requires permission:

The new module $M_{R}(a, 1, \varepsilon)$ is:

Wait for an expansionary stage, and then request permission. When permission is received, add $2^{-a-1}$ to $\alpha$; wait for another expansionary stage and return with $U_{M}=\emptyset$.

For $n>1$, the new module $M_{R}(a, n, \varepsilon)$ is:

Let $b$ be the least $b>a$ such that $2^{-b}<\varepsilon / 2$; let $\varepsilon^{\prime}=\varepsilon /(2(b+n-a))$.

(1) For $k=a+1, a+2, \ldots, b+n$, call $M_{R}\left(k, n-1, \varepsilon^{\prime}\right)$, and add the returned set into $U_{M}$.

(2) Add the current version of $\beta\left\lceil b\right.$ into $U_{M}$ and wait for a stage at which $\beta\lceil b$ changes.

(3) Wait for an expansionary stage, and then request permission. When permission is received, add $2^{-b-n-1}$ to $\alpha$. Wait for another expansionary stage and return $U_{M}$.

\footnotetext{
${ }^{8}$ Recall from [19] that a very strong array is a computable sequence $\left(I_{n}\right)$ of disjoint finite sets of natural numbers such that $\left|I_{n}\right|<\left|I_{n+1}\right|$ for all $n \in \mathbb{N}$.

${ }^{9}$ Recall from [19] that a c.e. set $D$ is $\left\langle F_{n}\right\rangle$-a.n.c. $\left(\left\langle F_{n}\right\rangle\right.$ array non-computable) if for each c.e. set $W$ there is some $k$ such that $W \cap F_{k}=D \cap F_{k}$.
} 
For every $R$ and $n$, we run $M_{R}\left(a, 2^{a+1}, 2^{-n}\right)$ on the $n^{\text {th }}$ interval $[a, c)$ assigned for $R$. We also let $\alpha\left(c_{n}\right)=1$ iff $n \in D$, where $c_{n}$ is the $n^{\text {th }}$ element of $C$. That's the construction.

Lemmas 4.5, 4.6, and 4.7 hold for the new modules as well, so there is no interaction between the requirements. If $\Gamma^{\beta}=\alpha$, then the $R$-modules do not get stuck waiting for expansionary stages. For such $R$ and every $n$ such that $W_{R} \uparrow F_{n}=D \uparrow F_{n}$, every request for permission by the $n^{\text {th }}$ module for $R$ is granted, so the $n^{\text {th }}$ module for $R$ gets stuck waiting for $\beta$ to leave $U_{n}$ (the open set enumerated by $R$ ). Thus for infinitely many $n$ we have $\beta \in U_{n}$. By adjusting the set (letting $U_{m}^{\prime}=\bigcup_{n>m} U_{n}$ ) we build a Martin-Löf test which covers $\beta$, so $\beta$ is not random.

4.4. Failure of multiple permitting II: Random reals. Here we show Proposition 1.11; If $\alpha$ has array computable Turing degree, then there is some random left-c.e. real $\beta \geqslant{ }_{\mathrm{ibT}} \alpha$.

Again the idea is to use an approximation to $\alpha$ which doesn't change too much, and build $\beta$ by requesting that it change by at least $2^{-n}$ whenever $\alpha\lceil n$ changes. On top of this, we need to ensure that $\beta$ is random. To accomplish this task, let $U$ be the first element of the universal Martin-Löf test. If we ensure that $\beta \notin U$, then we will have ensured that $\beta$ is random.

We can think of $U$ as a c.e. antichain of strings (the set of reals is the set of all infinite extensions of strings in $U$ ). So $\sum_{\sigma \in U} 2^{-|\sigma|} \leqslant 1 / 2$. To ensure that $\beta$ is not in $U$, whenever we discover that there is some $\sigma \in U$ which is an initial segment of the current version of $\beta$, we request that this change by adding $2^{-|\sigma|}$ to $\beta$. Thus if the approximation to $\alpha$ is sufficiently tight so that the total amount of quantity added to $\beta$ on behalf of following $\alpha$ is less than half, this strategy will succeed.

So fix a computable order which satisfies

$$
\sum_{n \geqslant 1} g(n) 2^{-n}<1 / 2
$$

(we allow the value 0 for $g$ for some finitely many inputs; we assume that if $g(n)=0$, then $\alpha\lceil n$ is simply given to us as a parameter in the construction).

Get a computable approximation $f_{s}(n)$ for the function $n \mapsto \alpha \uparrow n$ such that for all $n$,

$$
\#\left\{s: f_{s+1}(n) \neq f_{s}(n)\right\} \leqslant g(n) .
$$

The left-c.e. real $\beta$ is defined by recursion: we start with $\beta_{0}=0$ and let

$$
\beta_{s+1}=\beta_{s}+\sum\left\{2^{-n}: f_{s+1}(n) \neq f_{s}(n)\right\}+\sum\left\{2^{-|\sigma|}: \sigma \in U_{s} \& \sigma \subset \beta_{s}\right\} .
$$

Lemma 4.8. $\beta<1$ (and so is well defined as an element of $2^{\omega}$ ).

Proof. There are two kinds of contributions to $\beta$ : following $\alpha$ and avoiding $U$. Now $g$ satisfies $\sum_{n} g(n) 2^{-n}<1 / 2$ and for all $n$ we have $\#\left\{s: f_{s+1}(n) \neq f_{s}(n)\right\} \leqslant g(n)$, so the total added to $\beta$ by the clause $\sum\left\{2^{-n}: f_{s+1}(n) \neq f_{s}(n)\right\}$ is less than one-half. Because $\beta$ is a left-c.e. real, for every $\sigma \in U$, there is at most one stage $s$ at which we have $\sigma \in U_{s}$ and $\sigma \subset \beta_{s}$, so the contribution of the clause $\sum\left\{2^{-|\sigma|}: \sigma \in U_{s} \& \sigma \subset \beta_{s}\right\}$ is at most $\sum_{\sigma \in U} 2^{-|\sigma|} \leqslant 1 / 2$. 
Lemma 4.9. $\beta \notin U$.

Proof. First note that $\beta$ is not computable, because otherwise $\alpha$ would be computable. So $\beta$ is irrational, it has a unique infinite binary expansion and the digits of $\left(\beta_{s}\right)$ converge pointwise to the digits of $\beta$. If $\beta \in U$, then there is some $\sigma \in U$ such that $\sigma \subset \beta$. Then at a late enough stage $s$ we have $\sigma \in U_{s}$ and $\sigma \subset \beta_{s}$, so at stage $s$ we add $2^{-|\sigma|}$ to $\beta$ and force $\sigma \not \subset \beta$ forever.

Finally, the first clause in the definition of $\beta$ shows that $\beta \geqslant_{\mathrm{ibT}} \alpha$ (see Lemma 4.3).

\section{CL REDUCTIONS OUTSIDE THE C.E. DEGREES}

Here we prove Theorem 1.13, every Turing degree $\mathbf{d}$ which is not generalised $\mathrm{low}_{2}$ computes a real which is not cl-reducible to a random real. As discussed in the introduction, this is an application of non- $\mathrm{GL}_{2}$-permitting to a construction of Hirschfeldt's. The basic Lemma of Hirschfeldt (which can be found in [16]) is:

Lemma 5.1. If $h$ is a computable order and $\Gamma$ is an ibT-functional, then the set of strings $\tau \in 2^{<\omega}$ such that for all $\eta \in 2^{|\tau|}$, if $\Gamma^{\eta}=\tau$, then $K(\eta)<h(|\eta|)$, is dense.

Iterating Lemma 5.1 over all computable orders (since there is a $\mathbf{0}^{\prime}$-listing of those) and ibT-functionals, Hirschfeldt gets a real below $\mathbf{0}^{\prime}$ which is not cl-reducible to a complex real. An immediate consequence of Hirschfeldt's lemma is that every 2 -generic real is also not cl-reducible to a complex real. To get our result, we use the technical Lemma 5.2 to show that non- $\mathrm{GL}_{2}$ degrees provide sufficient genericity (compare with the fact that every non-GL $\mathrm{GL}_{2}$ degree bounds a 1-generic real).

Lemma 5.2. Suppose that $\left\langle D_{e}\right\rangle$ is a sequence of subsets of $2^{<\omega}$, uniformly computable from $\mathbf{0}^{\prime}$. Also suppose that $\left\{e: D_{e}\right.$ is dense $\}$ is $\Pi_{2}^{0}$-definable. Then every non-GL $L_{2}$ degree bounds a real which meets every set $D_{e}$ which is dense.

To obtain Theorem 1.13 from Lemma 5.2 let $\left\langle\Gamma_{e}\right\rangle$ be an effective listing of all ibT-functionals and let $\left\langle h_{i}\right\rangle$ be an effective listing of all non-decreasing, partial computable functions (whose domain is an initial segment of $\omega$ ) such that for all $i$, if $h_{i}$ is total, then it is unbounded, and so an order (such a listing can be obtained by not recognising subsequent convergences until a larger value is obtained). For $e, i<\omega$, let $D_{e, i}$ be the set of strings $\tau$ such that $h_{i}(m) \downarrow$ for all $m \leqslant|\tau|$ and for all $\eta \in 2^{|\tau|}$, if $\Gamma_{e}^{\eta}=\tau$, then $K(\eta)<h_{i}(|\tau|)$. These sets are uniformly computable in $\mathbf{0}^{\prime}$. If $h_{i}$ is total, then it is an order and so by Hirschfeldt's 5.1] $D_{e, i}$ is dense. If $h_{i}$ is not total, then $D_{e, i}$ is finite. It follows that the set of pairs $(e, i)$ such that $D_{e, i}$ is dense is $\Pi_{2}^{0}$-definable. By Lemma [5.2, if $\mathbf{d}$ is non-GL $L_{2}$, there is some $A \leqslant_{T} \mathbf{d}$ such that for all $e$ and $i$ such that $h_{i}$ is an order, there is some $\tau \subset A$ which is in $D_{e, i}$. Then if $B \geqslant{ }_{\mathrm{ibT}} A$ and $h$ is an order, then there is some $\tau \subset A$ such that $K(B \uparrow|\tau|)<h(|\tau|)$ and so $B$ is not complex via $h$; so $B$ is not complex.

We turn to prove Lemma 5.2. We define two fast-growing functions, both computable in $\mathbf{0}^{\prime}$ :

- Let $D_{e, s}$ be a computable approximation for $D_{e}$ (uniformly in $e$ ). Let $m_{e}$ be a modulus for $D_{e, s}$, uniformly computable from $\mathbf{0}^{\prime}$. Let $f_{0}$ be a function such that for all $n$, for all $e \leqslant n$ and all $\sigma \in 2^{\leqslant n}, f_{0}(n) \geqslant m_{e}(\sigma)$.

- The set of indices $e$ such that $D_{e}$ is dense is co-c.e. in $\mathbf{0}^{\prime}$. Using an enumeration of the set of $e$ 's such that $D_{e}$ is not dense, we can, with oracle 
$\mathbf{0}^{\prime}$, compute a function which takes a string $\sigma$ to an extension in $D_{e}$, or discovers that $D_{e}$ is not dense. So computably from $\mathbf{0}^{\prime}$ we can compute a function $f_{1}$ such that for all $n$, for all $e \leqslant n$ and all $\sigma \in 2^{\leqslant n}$, if $D_{e}$ is dense, then $f_{1}(n)$ bounds the length of some extension of $\sigma$ in $D_{e}$.

We may further assume that if $D_{e}$ is not dense, then it is finite; because as mentioned, $\mathbf{0}^{\prime}$ can enumerate those $e^{\prime}$ s such that $D_{e}$ is not dense, and once $e$ is enumerated, $\mathbf{0}^{\prime}$ can declare that no more strings are ever accepted as elements of $D_{e}$. Indeed, we may assume that if $D_{e}$ is not dense, then there is a stage $s$ such that $D_{e, s}$ is finite and for all $t \geqslant s, D_{e, t}=D_{e, s}$, because we can approximate these $e$ 's in a $\Sigma_{2}^{0}$ fashion and refuse to accept changes to $D_{e, t}$ until an "expansionary" stage is discovered. [In detail: say that $D_{e}$ is dense iff $\forall x \exists y C(e, x, y)$, where $C$ is a computable predicate. Inductively define e-expansionary stages to be 0 , and if $s$ is $e$-expansionary, then the next $e$-expansionary stage is the next stage $t$ such that for all $x<s$ there is some $y<t$ such that $C(e, x, y)$ holds. Redefine the approximation to $D_{e}$ by letting $\hat{D}_{e, t}=D_{e, s}$ for the greatest $e$-expansionary stage $s \leqslant t$.]

The functions $f_{0}$ and $f_{1}$ defined above have a property which is somewhat stronger than simply being $\Delta_{2}^{0}$ : by possibly increasing their values, they each have a computable approximation which is non-decreasing in $s$ (this doesn't change their desired properties). It follows that the function $f$, defined recursively by letting $f(0)=0$ and $f(s+1)=f_{0}\left(f_{1}(f(s))\right.$, also has such an approximation.

Lemma 5.3. There is some $g \leqslant_{T} \mathbf{d}$ such that for all $n, g(n) \leqslant f(n)$ and for infinitely many $n, g(n)=f(n)$.

Proof. Let $m_{f}$ be a modulus for $f_{s}$, the non-decreasing (in $s$ ) computable approximation for $f$. Since $\mathbf{d}$ is non-GL 2 , there is some $h \leqslant_{T} \mathbf{d}$ which is not dominated by $m_{f}$. For all $n$, let $g(n)=f_{h(n)}(n)$.

We use $g$ to bound the searches in our imitation of the $\mathbf{0}^{\prime}$ construction: we recursively define an increasing sequence of strings $\sigma_{s}$, starting with $\sigma_{0}=\langle\rangle$, aiming to define $A=\bigcup_{s} \sigma_{s}$ and try to meet the requirements

$R_{e}$ : There is some $\sigma \subset A$ in $D_{e}$.

At stage $s$, we say that requirement $R_{e}$ is currently satisfied if there is some $\tau \subseteq \sigma_{s}$ such that $\tau \in D_{e, g(s+1)}$. A requirement $R_{e}$ requires attention at stage $s$ if it is not currently satisfied and there is some $\tau \supset \sigma_{s}$ of length at most $g(s+1)$ which is in $D_{e, g(s+1)}$. At stage $s$ we act for the strongest requirement which wishes to act. To act for $R_{e}$, we choose the shortest extension $\tau$ of $\sigma_{s}$ which is in $D_{e, g(s+1)}$ and set $\sigma_{s+1}=\tau$. If no requirement wishes to act, we set $\sigma_{s+1}=\sigma_{s}$. That's the construction.

Lemma 5.4. For all $s,\left|\sigma_{s}\right| \leqslant g(s)$, and so for all $s,\left|\sigma_{s}\right| \leqslant f(s)$.

A stage $s$ is called true if $f(s+1)=g(s+1)$.

Lemma 5.5. Suppose that a requirement $R_{e}$ feels satisfied at a true stage $s>e$. Then it is met (and feels satisfied at every stage $t \geqslant s$ ).

Proof. At stage $s$ we identify some $\tau \subseteq \sigma_{s}$ which is in $D_{e, g(s+1)}=D_{e, f(s+1)}$. Since $f(s+1) \geqslant f_{0}(f(s))$ and $f(s) \geqslant|\tau|$, e, we have $f(s+1) \geqslant m_{e}(\tau)$ and so $\tau \in D_{e}$. For every $t \geqslant s$, since we may assume that $g(t+1) \geqslant g(s+1)$, we have $\tau \in D_{e, g(t+1)}$ and so $R_{e}$ feels satisfied at stage $t$. 
Lemma 5.6. Suppose that $D_{e}$ is dense and that requirement $R_{e}$ acts at a true stage $s>e$. Then $R_{e}$ is met and feels satisfied at every stage $t>s$.

Proof. At stage $s$ we define $\sigma_{s+1}=\tau$, where $\tau$ is the shortest extension of $\sigma_{s}$ in $D_{e, g(s+1)}=D_{e, f(s+1)}$. Since $D_{e}$ is dense and $\left|\sigma_{s}\right| \leqslant f(s)$, we know that there is an extension $\tau^{\prime}$ of $\sigma_{s}$ in $D_{e}$ of length at most $f_{1}(f(s))$, and that $f(s+1)=$ $f_{0}\left(f_{1}(f(s))\right) \geqslant m_{e}\left(\tau^{\prime}\right)$, so $\tau^{\prime} \in D_{e, f(s+1)}$. So $|\tau| \leqslant\left|\tau^{\prime}\right| \leqslant f_{1}(f(s))$ and so $f(s+1) \geqslant$ $m_{e}(\tau)$. So $\tau \in D_{e}$ and for all $t>s$ we have $\tau \in D_{e, g(t+1)}$ (again we assume that $g(t+1) \geqslant g(s+1)$, so $R_{e}$ feels satisfied at stage $t$.

Lemma 5.7. If $D_{e}$ is dense, $s>e$ is a true stage, and $R_{e}$ does not feel satisfied at stage $s$, then $R_{e}$ requires attention at stage $s$.

Proof. Since $D_{e}$ is dense, there is some extension of $\sigma_{s}$ in $D_{e}$. In fact, since $\left|\sigma_{s}\right| \leqslant$ $f(s)$, there is such an extension $\tau$ of length at most $f_{1}(f(s))$, and so $g(s+1)=$ $f(s+1)=f_{0}\left(f_{1}(f(s))\right) \geqslant m_{e}(\tau)$, so $\tau \in D_{e, g(s+1)}$, so $R_{e}$ requires attention at stage $s$.

Lemma 5.8. If $D_{e}$ is not dense, then there is a stage after which $R_{e}$ never requires attention.

Proof. If $n$ is the greatest length of a string which appears in any $D_{e, s}$, then after stage $n, R_{e}$ never finds an extension of $\sigma_{n}$ in $D_{e, g(n+1)}$ and so does not require attention.

Overall we see that every requirement eventually stops requiring attention and so the finite-injury priority argument works and for all $e$ such that $D_{e}$ is dense, $R_{e}$ is met. This concludes the proof of Lemma 5.2 .

Remark 5.9. Lemma 5.2 holds if the set of dense sets is only $\Sigma_{3}^{0}$-definable. This can be seen by a modification of the proof, but also directly using the lemma for $\Pi_{2}^{0}$ and changing the enumeration of the sets: change $\left\langle D_{e}\right\rangle$ to $\left\langle D_{e, t}\right\rangle$, where $D_{e, t}=D_{e}$ if $t$ is a $\Sigma_{3}^{0}$-witness for the fact that $D_{e}$ is dense, and otherwise make $D_{e, t}$ finite.

Remark 5.10. Another way to show Lemma 5.2 was pointed out by the anonymous referee. Let $D_{e}$ be as in Lemma 5.2 and let $h(e, n)$ be a computable 0-1 function such that $D_{e}$ is dense iff $f(e, n)=1$ for infinitely many $n$. The sets

$$
C_{e}=D_{e} \cup\{\sigma|| \sigma \mid>n \text { and } f(e, m)=0 \text { for all } m>n\}
$$

are uniformly $\emptyset^{\prime}$-computable and dense. Also, if $D_{e}$ is dense, then $C_{e}=D_{e}$, so meeting every $C_{e}$ is the same as meeting all dense $D_{e}$.

Thus Lemma 5.2 reduces to showing that for every uniformly $\emptyset^{\prime}$-computable sequence of dense sets of binary strings, every non- $\mathrm{GL}_{2}$ degree bounds a real meeting every set in the sequence. This is a consequence of a combination of results in computable model theory and genericity arguments with $\mathrm{GL}_{2}$ from [20, 9].

\section{Proof of a theorem of Frank Stephan}

We give a proof of Theorem 1.12 (due to Frank Stephan) that the left-c.e. random reals inhabit more than one cl-degree. First we give some immunity properties of random sequences.

Definition 6.1 (Fenner and Schaefer [22]). A set $A$ is called $k$-immune $(k \in \mathbb{N})$ if there is no computable sequence $\left\langle F_{n}\right\rangle$ of pairwise disjoint sets such that for all $n$, $\left|F_{n}\right| \leqslant k$ and $F_{n} \cap A \neq \emptyset$. 
Note that every set is 0-immune and that the 1-immune sets are exactly the immune sets; also, hyperimmune sets are $k$-immune for all $k<\omega$. Fenner and Schaefer [22] showed that the classes of $k$-immune sets form a proper hierarchy.

Theorem 6.2 (Folklore).

(1) For all $k<\omega$, every random set is $k$-immune (indeed, every Kurtz-random 10 set is k-immune).

(2) No random set is hyperimmune.

Proof. For (1) we need the following.

Lemma 6.3. Suppose that $|B|=m$ and that $\mathcal{B} \subseteq \mathcal{P}(B)$ is a collection of $n$ pairwise disjoint subsets of $B$, each of size $k$. Then there are exactly $2^{m-k n}\left(2^{k}-1\right)^{n}$ subsets of $B$ which have non-empty intersection with every set in $\mathcal{B}$.

Proof. Each $B^{\prime} \in \mathcal{B}$ has $2^{k}-1$ non-empty subsets; so there are $\left(2^{k}-1\right)^{n}$ many subsets of $\bigcup \mathcal{B}$ with non-empty intersection with every $B^{\prime} \in \mathcal{B}$. The size of $B-\cup \mathcal{B}$ is $m-k n$; each subset of $B$ of the desired property is determined by its intersection with $\bigcup \mathcal{B}$ and with $B-\bigcup \mathcal{B}$, which can be chosen independently.

For (1), let $\left\langle F_{n}\right\rangle$ be a computable collection of pairwise disjoint sets, each of size $k$, and let $\mathcal{Q}$ be the class of sets which have non-empty intersection with every $F_{n}$. Then $\mathcal{Q}$ is $\Pi_{1}^{0}$. By weeding, we may assume that for all $n, \max F_{n}<\min F_{n+1}$. By Lemma 6.3. for every $n$, The number of subsets of $1+\max F_{n}$ which intersect every $F_{m}$ (for $\left.m=1, \ldots, n\right)$ is $2^{1+\max F_{n}-k n}\left(2^{k}-1\right)^{n}$ and so the measure of the class $\mathcal{Q}_{n}$ of infinite sets which intersect every $F_{m}$ for $m=1, \ldots, n$ is

$$
\mu\left(\mathcal{Q}_{n}\right)=\frac{2^{1+\max F_{n}-k n}\left(2^{k}-1\right)^{n}}{2^{1+\max F_{n}}}=\left(1-\frac{1}{2^{k}}\right)^{n},
$$

which approaches 0 as $1-2^{-k}<1$. Since $\mathcal{Q}=\bigcap \mathcal{Q}_{n}, \mathcal{Q}$ is a null class. So if $A$ is Kurtz-random, then $A \notin \mathcal{Q}$, so $A$ doesn't fail to be $k$-immune because of $\left\langle F_{n}\right\rangle$.

For (2), let $\left\langle F_{n}\right\rangle$ be a computable sequence of pairwise disjoint sets such that $\left|F_{n}\right|=2^{n}$. This time let $\mathcal{Q}_{n}$ be the class of sets $A$ such that $F_{n} \cap A$ is empty. So $\mu\left(\mathcal{Q}_{n}\right)=2^{-n}$. So if we let $U_{n}=\bigcup_{m>n} \mathcal{Q}_{n}$, then $\left\langle U_{n}\right\rangle$ is a Martin-Löf test, and $\bigcap_{n} U_{n}$ is the collection of sets $A$ such that there are infinitely many $n$ such that $F_{n} \cap A$ is empty, and so contains every hyperimmune set.

Proof of Theorem 1.12. Let $\alpha$ be a random left-c.e. real. Note that $\omega \backslash \alpha$ (where $\alpha$ is viewed as a subset of $\omega$ ) is also random. By Theorem 6.2(2), there is a strictly increasing computable sequence $\left\langle t_{n}\right\rangle$ such that for every $n, \alpha \uparrow\left(t_{n}, t_{n+1}\right]$ contains a 0 digit.

Let $\beta(x)=1$ iff $x=t_{n}$ for some $n>0$ (so $\beta=\sum_{n>0} 2^{-t_{n}-1}$ ). For every $n>0$,

$$
\alpha \uparrow\left(t_{n}+1\right)+\beta \uparrow\left(t_{n}+1\right)=(\alpha+\beta) \uparrow\left(t_{n}+1\right) ;
$$

this is because for $m<n, \alpha \uparrow\left(t_{m}, t_{m+1}\right]$ is not a string of 1 's and so adding $2^{-t_{m}-1}$ doesn't carry before the $t_{m}{ }^{\text {th }}$ digit. It follows that

$$
K\left((\alpha+\beta) \uparrow t_{n}+1\right)={ }^{+} K\left(\alpha \uparrow t_{n}+1\right)={ }^{+} t_{n}
$$

(here $={ }^{+}$denotes equality modulo a constant) and so $\alpha+\beta$ is random (and left-c.e.).

\footnotetext{
${ }^{10}$ Recall from e.g. 32 that a set is Kurtz-random iff it is not a member of a null $\Pi_{1}^{0}$ class. This is a very weak notion of randomness.
} 
We claim that $\alpha$ does not cl-compute $\alpha+\beta$. For otherwise there would be some constant $c<\omega$ and a partial computable function $\psi$ such that for all $n$,

$$
\psi(\alpha \uparrow(n+c))=(\alpha+\beta)(n) .
$$

By Theorem 6.2(1), there are infinitely many $n$ such that $\alpha \uparrow\left[t_{n}-c, t_{n}\right)=1^{c}$. For each such $n$, by Equation 6.1, we have

$$
\psi\left(\alpha \uparrow t_{n}\right)=(\alpha+\beta)\left(t_{n}-c\right)=1-\alpha\left(t_{n}\right) .
$$

But this gives rise to a c.e. martingale 11 which succeeds on $\alpha$ : let $M(\sigma i)$ split $M(\sigma)$ evenly between both outcomes, except when $|\sigma|=t_{n}$ for some $n>0$ and $\sigma$ ends with a string of $c 1$ 's; then wait for $\psi(\sigma)$ to converge, in which case all the capital is spent guessing the next bit is $1-\psi(\sigma)$. This, of course, contradicts the fact that $\alpha$ is random.

\section{REFERENCES}

[1] Bahareh Afshari, George Barmpalias, S. Barry Cooper and Frank Stephan, Post's programme for the Ershov hierarchy, Journal of Logic and Computation 17 (2007), 1025-1040. MR2376073 (2008j:03059)

[2] George Barmpalias, Computability and applications to analysis, Ph.D. thesis, University of Leeds, U.K., 2004.

[3] George Barmpalias, Hypersimplicity and semicomputability in the weak truth table degrees, Archive for Math. Logic 44 (2005), 1045-1065. MR2193189 (2006m:03069)

[4] George Barmpalias and Andrew E. M. Lewis, Random reals and Lipschitz continuity, Mathematical Structures in Computer Science, Volume 16 (2006), 737-749. MR2268340 (2007h:03086)

[5] George Barmpalias and Andrew E. M. Lewis, The ibT degrees of computably enumerable sets are not dense, Annals of Pure and Applied Logic, Volume 141 (2006), 51-60. MR2229929 (2007d:03068)

[6] George Barmpalias and Andrew E. M. Lewis, Randomness and the linear degrees of computability, Annals of Pure and Applied Logic 145 (2007), 252-257. MR2286414|(2007j:03056)

[7] George Barmpalias and Andrew E. M. Lewis, A c.e. real that cannot be sw-computed by any $\Omega$-number, Notre Dame Journal of Formal Logic, Volume 47 Issue 2 (2006), pp. 197-209. MR2240619 (2008c:03046)

[8] John Chisholm, Jennifer Chubb, Valentina S. Harizanov, Denis R. Hirschfeldt, Carl G. Jockusch, Jr., Timothy McNicholl, and Sarah Pingrey, $\Pi_{1}^{0}$ Classes and strong degree spectra of relations, Journal of Symbolic Logic 72 (2007), 1003-1018. MR2354911

[9] Chris J. Conidis, Classifying Model-Theoretic Properties, Journal of Symbolic Logic, Vol. 73(3) (2008) 885-905. MR2444274

[10] Barbara F. Csima and Robert I. Soare, Computability results used in differential geometry, Journal of Symbolic Logic 71 (2006), 1394-1410. MR.2275866 (2007g:03053)

[11] David Doty, Every sequence is decompressible from a random one. in Arnold Beckmann et al. (eds.), Logical approaches to computational barriers, Proceedings of the second conference on computability in Europe, Lecture Notes in Computer Science 3988, Springer-Verlag, 2006, $153-162$.

\footnotetext{
${ }^{11}$ Martingales are another way to define effective randomness. Recall, e.g. from [32, that a martingale is a function $d$ from binary strings to non-negative reals such that $2 d(\sigma)=d(\sigma 0)+d(\sigma 1)$ for all strings $\sigma$. A martingale is computably enumerable (c.e.) if there is a computable procedure which, given $\sigma$, produces a computable increasing sequence of rationals converging to $d(\sigma)$. We say that a martingale succeeds on an infinite binary sequence if the supremum of the values it takes on the sequence is unbounded. It is a basic fact that a sequence $Z$ is Martin-Löf random iff no c.e. martingale succeeds on $Z$.
} 
[12] Rod Downey and Noam Greenberg, Totally $<\omega^{\omega}$-computably enumerable degrees and embeddings of the 1-3-1 lattice, in preparation.

[13] Rod Downey and Noam Greenberg, Totally $<\omega^{\omega}$-computably enumerable and $m$-topped degrees, In Cai, Cooper, Li (eds.), Theory and Applications of Models of Computation (proceedings of TAMC 2006, Beijing), volume 3959 of Lecture Notes in Computer Science, Springer (2006), 46-60. MR2277228 (2007i:03047)

[14] Rod Downey and Noam Greenberg, Turing degrees of reals of positive effective packing dimension, Information Processing Letters 108 (2008), 298-303. MR2456604

[15] Rod Downey, Noam Greenberg and Rebecca Weber, Totally $\omega$-computably enumerable degrees and bounding critical triples, J. Math. Log. 7 (2007), 145-171. MR2423948

[16] Rod Downey and Denis R. Hirschfeldt, Algorithmic randomness and complexity, Springer, to appear.

[17] Rod Downey, Denis R. Hirschfeldt and Geoff LaForte, Randomness and reducibility. Mathematical foundations of computer science, 2001, 316-327, Lecture Notes in Comput. Sci. 2136, Springer, Berlin, 2001. MR 1907022 (2003j:03051)

[18] Rod Downey, Denis R. Hirschfeldt and Geoff LaForte, Randomness and reducibility. Journal of Computer and System Sciences 68 (2004), 96-114. MR2030512 (2004m:03165)

[19] Rod Downey, Carl G. Jockusch, Jr., and Michael Stob, Array nonrecursive sets and multiple permitting arguments, Recursion Theory Week (Proceedings, Oberwolfach 1989) (K. AmbosSpies, G.H. Müller and G.E. Sacks, eds.) Lecture Notes in Math. 1432, Springer-Verlag, 1990, 141-173. MR:1071516 (91k:03110)

[20] Denis R. Hirschfeldt, Richard A. Shore, and Theodore A. Slaman, The atomic model theorem, Preprint.

[21] Rod Downey, Carl G. Jockusch, Jr., and Michael Stob, Array nonrecursive sets and genericity, in Computability, Enumerability, Unsolvability: Directions in Recursion Theory. London Mathematical Society Lecture Notes Series (Cambridge University Press) 224 (1996), 93104. MR1395876 (97f:03060)

[22] Stephen Fenner and Marcus Schaefer, Bounded immunity and btt-reductions. Mathematical Logic Quarterly 45 (1999), 3-21. MR1669934 (2000d:03087)

[23] Peter Gács, Every sequence is reducible to a random one, Information and Control 70 (1986), 186-192. MR859105 (87k:03043)

[24] Shamil Ishmukhametov, Weak recursive degrees and a problem of Spector, in Recursion Theory and Complexity (M. Arslanov and S. Lempp, eds.), de Gruyter, Berlin, 1999, 81-88. MR.1724934 (2001a:03089)

[25] Bjørn Kjos-Hanssen, Wolfgang Merkle and Frank Stephan, Kolmogorov complexity and the recursion theorem, in STACS 2006: Twenty-Third Annual Symposium on Theoretical Aspects of Computer Science, Marseille, France, February 23-25, 2006. Proceedings. Springer Lecture Notes in Computer Science 3884, 149-161, 2006. MR2249365 (2008a:68100)

[26] Antonin Kučera, Measure, $\Pi_{1}^{0}$-classes and complete extensions of PA. In: H.-D. Ebbinghaus et al. (eds.), Recursion Theory Week. Lecture Notes in Mathematics 1141:245-259, Springer, 1985. MR820784 (87e:03102)

[27] Antonin Kučera, On the use of diagonally nonrecursive functions. In: H.-D. Ebbinghaus et al. (eds.), Logic Colloquium 198\%. Studies in Logic and the Foundations of Mathematics 129:219-239, North-Holland, 1989. MR1015310 (91c:03037)

[28] Antonin Kučera and Theodore A. Slaman, Randomness and recursive enumerability. SIAM Journal of Computing 31 (2001), 199-211. MR.1857396 (2002k:68078)

[29] Martin Kummer, Kolmogorov complexity and instance complexity of recursively enumerable sets, SIAM Journal on Computing 25 (1996), No. 6, 1123-1143. MR1417892 (98h:03055)

[30] M. Li and P. Vitányi, An introduction to Kolmogorov complexity and its applications, Graduate Texts in Computer Science, Second Edition, Springer-Verlag, New York, 1997. MR:1438307 (97k:68086)

[31] Wolfgang Merkle and Nenad Mihailović, On the construction of effectively random sets, Journal of Symbolic Logic 69 (2004), 862-878. MR2078927 (2005k:03103)

[32] André Nies. Computability and Randomness. Oxford University Press, in preparation, 2009.

[33] Keng Meng Ng, Frank Stephan and Guohua Wu, Degrees of weakly computable reals, Proceedings of "Second Conference on Computability in Europe", CiE 2006, Swansea (2006), $413-422$. 
[34] Piergiorgio Odifreddi, Classical recursion theory, Volume I, North-Holland Publ. Co., Amsterdam, 1989. MR.982269 (90d:03072)

[35] Liang Yu and Decheng Ding, There is no $S W$-complete c.e. real. J. Symbolic Logic 69 (2004), 1163-1170. MR2135660 (2006h:68075)

School of Mathematics, Statistics and Computer Science, Victoria University, P.O. Box 600, Wellington, New Zealand

E-mail address: George.Barmpalias@mcs.vuw.ac.nz

School of Mathematics, Statistics and Computer Science, Victoria University, P.O. Box 600, Wellington, New Zealand

E-mail address: downey@mcs.vuw.ac.nz

School of Mathematics, Statistics and Computer Science, Victoria University, P.O. Box 600, Wellington, New Zealand

E-mail address: greenberg@mcs.vuw.ac.nz 